\title{
Scalar curvature and the multiconformal class of a direct product Riemannian manifold
}

\author{
Saskia $\operatorname{Roos}^{1}$ (D) Nobuhiko Otoba ${ }^{2}$
}

Received: 7 November 2019 / Accepted: 25 June 2021 / Published online: 6 July 2021

(c) The Author(s) 2021

\begin{abstract}
For a closed, connected direct product Riemannian manifold $(M, g)=\left(M_{1}, g_{1}\right) \times \cdots \times$ $\left(M_{l}, g_{l}\right)$, we define its multiconformal class $\llbracket g \rrbracket$ as the totality $\left\{f_{1}^{2} g_{1} \oplus \cdots \oplus f_{l}^{2} g_{l}\right\}$ of all Riemannian metrics obtained from multiplying the metric $g_{i}$ of each factor $M_{i}$ by a positive function $f_{i}$ on the total space $M$. A multiconformal class $\llbracket g \rrbracket$ contains not only all warped product type deformations of $g$ but also the whole conformal class $[\tilde{g}]$ of every $\tilde{g} \in \llbracket g \rrbracket$. In this article, we prove that $\llbracket g \rrbracket$ contains a metric of positive scalar curvature if and only if the conformal class of some factor $\left(M_{i}, g_{i}\right)$ does, under the technical assumption $\operatorname{dim} M_{i} \geq 2$. We also show that, even in the case where every factor $\left(M_{i}, g_{i}\right)$ has positive scalar curvature, $\llbracket g \rrbracket$ contains a metric of scalar curvature constantly equal to -1 and with arbitrarily large volume, provided $l \geq 2$ and $\operatorname{dim} M \geq 3$.
\end{abstract}

Keywords Positive scalar curvature - Constant scalar curvature - The Yamabe problem . Warped product $\cdot$ Umbilic product . Twisted product

Mathematics Subject Classification (2010) Primary 53C21

\section{Contents}

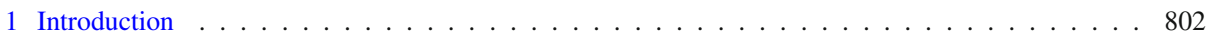

2 The normalized Einstein-Hilbert functional . . . . . . . . . . . . . . . . . . . . . 803

3 The scalar curvature of a multiconformal class . . . . . . . . . . . . . . . . . . . . . 806

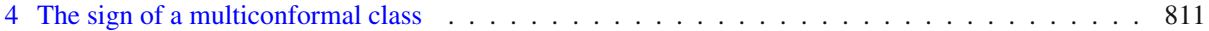

5 The infimum of the conformal Yamabe invariants . . . . . . . . . . . . . . . . . . . . 813

6 Multiconformal metric of permutation type . . . . . . . . . . . . . . . . . . . . . 815

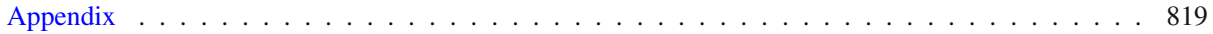

A Computation of the scalar curvature . . . . . . . . . . . . . . . . . . . . . . 819

References . . . . . . . . . . . . . . . . . . . . . . . . 827

Saskia Roos

roos@math.uni-potsdam.de

Nobuhiko Otoba

nobuhiko.otoba@ur.de

1 Institut für Mathematik, Universität Potsdam, 14476 Potsdam, Germany

2 Universität Regensburg, 93040 Regensburg, Germany 
Table 1 Warped product metrics of constant scalar curvature

\begin{tabular}{llll}
\hline & $R^{g_{2}}>0$ & $R^{g_{2}}=0$ & $R^{g_{2}}<0$ \\
\hline$R^{g_{1}} \geq 0, R^{g_{1}} \not \equiv 0$ & $R^{\tilde{g}}>0$ & $R^{\tilde{g}}>0$ & \\
$R^{g_{1}} \equiv 0$ & $R^{\tilde{g}}>0$ & $R^{\tilde{g}}=0$ & $R^{\tilde{g}}<0$ \\
$\int_{M_{1}} R^{g_{1}} d \mu^{g_{1}} \leq 0, R^{g_{1}} \not \equiv 0$ & & $R^{\tilde{g}}<0$ & $R^{\tilde{g}}<0$ \\
\hline
\end{tabular}

\section{Introduction}

Let $M$ be a closed connected $m$-dimensional manifold and let $[g]$ be the conformal class of a Riemannian metric $g$. The conformal Yamabe invariant $\mu(M,[g])$ is defined as

$$
\mu(M,[g])=\inf _{\tilde{g} \in[g]} \frac{\int_{M} R^{g} d \mu^{\tilde{g}}}{\operatorname{Vol}(M, \tilde{g})^{\frac{m-2}{m}}}>-\infty
$$

if $m \geq 3$ and as $\mu(M,[g])=4 \pi \chi(M)$ if $m=2$. By the resolution of the Yamabe problem (cf. $[3,25,38,45,52])$ this infimum is always attained by some metric $\tilde{g}$ of constant scalar curvature. In particular, each Riemannian metric $g$ on a closed manifold $M$ can be conformally changed into a metric of constant scalar curvature.

Next we consider a direct product $\left(M_{1}, g_{1}\right) \times\left(M_{2}, g_{2}\right)$ of two closed Riemannian manifolds and want to know how the metric $g_{1} \oplus g_{2}$ can be deformed into a metric of constant scalar curvature. Of course, we can conformally change each factor separately to obtain a metric of constant curvature. In that case, the sign of the resulting scalar curvature is in a direct relation with the signs of the conformal Yamabe invariants $\mu\left(M_{i},\left[g_{i}\right]\right)$ for $i=1,2$. Another common family of product manifolds are warped products $\left(M_{1} \times M_{2}, g_{1} \oplus f^{2} g_{2}\right)$, where $f: M_{1} \rightarrow \mathbb{R}_{+}$is a positive function.

In [12] the authors showed that if a warped product metric $\tilde{g}=g_{1} \oplus f^{2} g_{2}$ has constant scalar curvature then the scalar curvature $R^{g_{2}}$ has to be constant. Moreover, the sign of the scalar curvature $R^{\tilde{g}}$ is in many cases determined by sign of the scalar curvatures of $g_{1}$ and $g_{2}$. We summarize the known sign restrictions and their implication in Table 1, below.

Taking the product of constant scalar curvature metrics, we see that each case in Table 1 is nonempty and that, rescaling the metric $g_{2}$ by constants, there is no sign restriction on the scalar curvature in the two remaining cases in Table 1 that are left empty. The scalar curvature of warped product type metrics are also studied in [13-18,27,44,53].

We introduce the following perspective that unifies the previous sign restrictions on scalar curvature. For a direct product Riemannian manifold $(M, g)=\left(M_{1} \times \cdots \times M_{l}, g_{1} \oplus \cdots \oplus g_{l}\right)$, we define its multiconformal class $\llbracket g \rrbracket$ by

$$
\llbracket g \rrbracket:=\left\{f_{1}^{2} g_{1} \oplus \cdots \oplus f_{l}^{2} g_{l} \mid f_{1}, \ldots, f_{l}: M \rightarrow \mathbb{R}_{+}\right\} .
$$

Within a multiconformal class we may conformally change the metrics $g_{1}, \ldots, g_{l}$ respectively and take warped product metrics of all kind. Moreover, if $\tilde{g} \in \llbracket g \rrbracket$ then the whole conformal class $[\tilde{g}]$ belongs to $\llbracket g \rrbracket$. Our main result is the following trichotomy, see Remark 4.1 regarding the dimensional assumption.

Theorem 1.1 Let $(M, g)=\left(M_{1}, g_{1}\right) \times \cdots \times\left(M_{l}, g_{l}\right)$ be a direct product of closed connected Riemannian manifolds with $\operatorname{dim}\left(M_{i}\right) \geq 2$ for all $1 \leq i \leq l$. Then the following trichotomy holds.

(1) The multiconformal class $\llbracket g \rrbracket$ contains a metric of positive scalar curvature if and only if there exists $i \in\{1, \ldots, l\}$ such that $\mu\left(M_{i},\left[g_{i}\right]\right)>0$. 
(2) The multiconformal class $\llbracket g \rrbracket$ does not contain a metric of positive scalar curvature and there exists a scalar flat metric of $\llbracket g \rrbracket$ if and only if $\mu\left(M_{i},\left[g_{i}\right]\right)=0$ for every $i \in\{1, \ldots, l\}$. In this case, if $\tilde{g} \in \llbracket g \rrbracket$ has nonnegative scalar curvature, then $\tilde{g}$ is necessarily scalar flat and direct product.

(3) The multiconformal class $\llbracket g \rrbracket$ does not contain a metric of nonnegative scalar curvature if and only if for every $i \in\{1, \ldots, l\}, \mu\left(M_{i},\left[g_{i}\right]\right) \leq 0$ and there exists an $i \in\{1, \ldots, l\}$ such that $\mu\left(M_{i},\left[g_{i}\right]<0\right)$.

In particular, if $\mu\left(M_{i},\left[g_{i}\right]\right) \leq 0$ for all $1 \leq i \leq l$, then $\sup _{[\tilde{g}] \subset \llbracket g \rrbracket} \mu(M,[\tilde{g}])$ has to be nonpositive. But there is no similar result for the infimum. In fact, we show that within the multiconformal class $\llbracket g \rrbracket$ we can always find a metric with constant scalar curvature equal to -1 but with arbitrarily large volume. This is even the case when $\mu\left(M_{i},\left[g_{i}\right]\right)$ is nonnegative for each $1 \leq i \leq l$. However, in that case, such a metric of strictly negative scalar curvature cannot be of warped product type.

Theorem 1.2 Let $(M, g)=\left(M_{1}, g_{1}\right) \times \cdots \times\left(M_{l}, g_{l}\right)$ be a direct product of closed connected Riemannian manifolds with $l \geq 2$ and $\operatorname{dim}(M) \geq 3$, then

$$
\inf _{[\tilde{g}] \subset \llbracket g \rrbracket} \mu(M,[\tilde{g}])=-\infty .
$$

This article is organized as follows. In Sect. 2, we characterize criticality with respect to the normalized Einstein-Hilbert functional restricted to a multiconformal class. In Sect. 3, we compute the change of the scalar curvature under a multiconformal change and derive an integral formula which plays a crucial role in the proofs of Theorems 1.1 and 1.2. The trichotomy theorem (Theorem 1.1) is proved in Sect. 4. Afterwards, we prove Theorem 1.2 in Sect. 5. In Sect. 6, we discuss the structure of constant scalar curvature metrics within a multiconformal class.

\section{The normalized Einstein-Hilbert functional}

Let $M$ be a closed connected $m$-dimensional manifold with $m \geq 3$ and denote by Met the space of all Riemannian metrics on $M$. We would like to know whether there are $M$ admits metrics with special curvature conditions, e.g. Einstein metrics or metrics of constant curvature. One possible approach is to look for critical points of the normalized EinsteinHilbert functional

$$
\begin{aligned}
E: \text { Met } & \rightarrow \mathbb{R}, \\
g & \mapsto E(g):=\frac{\int_{M} R^{g} d \mu^{g}}{\operatorname{Vol}(M, g)^{\frac{m-2}{m}},}
\end{aligned}
$$

where $R^{g}$ denotes the scalar curvature of $g$. To characterize the critical points of $E$ we take a look on the variation formula for $E$. A straight-forward calculation shows that

$$
\left.\frac{d}{d t}\right|_{t=0} E(g+t h)=\int_{M}\left\langle h,\left(2^{-1} R^{g}-p_{m}^{-1} r^{g}\right) g-\operatorname{Ric}^{g}\right\rangle d \mu^{g},
$$

for each $h \in \Gamma\left(\operatorname{Sym}^{2} T^{*} M\right)$, where $r^{g}=\frac{\int_{M} R^{g} d \mu^{g}}{\operatorname{Vol}(M)}$. Therefore we see that $g$ is a critical value of $E$ if and only if $g$ is an Einstein metric. However, if we restrict $E$ to a conformal 
class $[g]$ the critical points are those metrics of constant scalar curvature conformal to $g$. In particular, if $g$ is a metric of constant scalar curvature the above formula simplifies to

$$
\left.\frac{d}{d t}\right|_{t=0} E(g+t h)=-\int_{M}\left\langle h, \operatorname{Ric}^{g}-\left(R^{g} / m\right) g\right\rangle d \mu^{g} .
$$

Aside critical points, we are also interested in the extreme values of the normalized Einstein-Hilbert functional itself. It is well-known that both, the infimum and supremum of $E:$ Met $\rightarrow \mathbb{R}$ is infinite. Thus, it is more convenient to look at the extreme values of restrictions of $E$, i.e. $E$ restricted to a conformal class $[g]$. While the supremum is still infinite, its infimum is well-known as the conformal Yamabe constant,

$$
\mu(M,[g])=\inf _{\tilde{g} \in[g]} E(\tilde{g}) .
$$

By the resolution of the Yamabe problem each $g \in$ Met is conformal tp a metric $\tilde{g}$, such that $E(\tilde{g})=\mu(M,[g])$ (cf. $[3,25,38,45,52])$. Such a metric $\tilde{g}$ is called a Yamabe metric. Each Yamabe metric has constant scalar curvature. Moreover, the supremum of all Yamabe constants on a manifold, is bounded from above by

$$
\sigma(M):=\sup _{[g] \in \mathrm{Met}} \mu(M,[g]) \leq \sigma\left(S^{m}\right)=m(m-1) \operatorname{Vol}\left(S^{m}(1)\right)^{\frac{2}{m}} .
$$

with equality if and only if $M$ is diffeomorphic to the sphere, [38].

In the following, we introduce two subspaces of metrics leading to two intermediate invariants between $\mu(M,[g])$ and $\sigma(M)$.

Definition 2.1 An almost product manifold $M$ is a manifold with a fixed decomposition of $T M=\bigoplus_{i=1}^{l} E_{i}$ into subbundles. A Riemannian metric $g$ on an almost product manifold $M$ is compatible if $E_{i} \perp E_{j}$ for all $i \neq j$ with respect to $g$. We denote by Met $\perp$ the space of all compatible metrics.

Note that each $g \in \mathrm{Met}_{\perp}$ uniquely decomposes as $g=g_{1} \oplus \cdots \oplus g_{l}$, where each $g_{i}$ is a bundle metric on $E_{i}$. This decomposition allows us to define the following equivalent relation on $\mathrm{Met}_{\perp}$ : We call two metrics $g, \tilde{g} \in \mathrm{Met}_{\perp}$ multiconformally equivalent if there are functions $f_{1}, \ldots, f_{l}: M \rightarrow \mathbb{R}_{+}$such that $\tilde{g}_{i}=f_{i}^{2} g_{i}$ for all $1 \leq i \leq l$. The corresponding equivalence class for a metric $g \in \mathrm{Met}_{\perp}$ is the multiconformal class $\llbracket g \rrbracket$. By construction, we have for each compatible metric $g$ the inclusions

$$
[g] \subset \llbracket g \rrbracket \subset \mathrm{Met}_{\perp} \subset \mathrm{Met} .
$$

For an almost product manifold $M$ we define invariants in the spirit of $\sigma(M)$ as follows

$$
\begin{aligned}
\sigma_{\perp}(M) & :=\sup _{[g] \subset \operatorname{Met}_{\perp}} \mu(M,[g]), \\
\sigma(M, \llbracket g \rrbracket) & :=\sup _{[\tilde{g}] \subset \llbracket g \rrbracket} \mu(M,[\tilde{g}]) .
\end{aligned}
$$

It is immediate that for any conformal class $[g] \subset \operatorname{Met}_{\perp}$

$$
-\infty<\mu(M,[g]) \leq \sigma(M, \llbracket g \rrbracket) \leq \sigma_{\perp}(M) \leq \sigma(M) \leq \sigma\left(S^{m}\right) .
$$

Lemma 2.2 Let $M$ be a closed connected almost product manifold of dimension $m \geq 3$. Then any of the invariants $\mu(M,[g]), \sigma(M, \llbracket g \rrbracket), \sigma_{\perp}(M)$ and $\sigma(M)$ is strictly positive if and only if $[g], \llbracket g \rrbracket, \mathrm{Met}_{\perp}$ and Met contain a metric of positive scalar curvature respectively. 
Proof Let $g$ be a metric on $M$ (not necessarily compatible). Then it follows from the resolution of the Yamabe problem that there exists a metric $\tilde{g} \in[g]$ of constant scalar curvature such that $E(\tilde{g})=\mu(M,[g])$. In fact, assuming without loss of generality that $\operatorname{Vol}(M, \tilde{g})=1$ it follows that $R^{\tilde{g}}=\mu(M,[g])$. Thus, $\mu(M,[g])>0$ if and only if $[g]$ contains a constant positive scalar curvature metric.

Since $\sigma(M, \llbracket g \rrbracket), \sigma\left(M, \mathrm{Met}_{\perp}\right)$ and $\sigma(M)$ are just supremum of conformal Yamabe invariants over different subsets of Met. Assuming one of them to be strictly positive implies that there has to be a conformal class $[g]$ in the respective subset of Met with $\mu(M,[g])>0$. Therefore, the lemma follows directly from the arguments given above.

By definition $\sigma(M, \llbracket g \rrbracket)$ and $\sigma_{\perp}(M)$ are invariants of almost product manifolds respectively. They have a resemblance in spirit to the equivariant Yamabe constant or invariant (cf. $[4,23]$ ) defined for manifolds with group actions. However, since an equivariant conformal class is smaller than the ordinary conformal class, one cannot expect an inequality like (2.3) for the equivariant ones (cf. [2, Example 3]).

If the invariants $\sigma(M, \llbracket g \rrbracket)$ or $\sigma_{\perp}(M)$ are attained for some metric $g \in$ Met $_{\perp}$ then it does not necessarily follow that $g$ is a critical value of the normalized Einstein-Hilbert functional $E$ restricted to $\llbracket g \rrbracket$ or $M_{\perp} t_{\perp}$ respectively. Indeed, we show below that the critical values of $E$ restricted to one of these subspaces have special curvature properties that lie between constant scalar curvature and being Einstein.

Let $M$ be a closed and connected almost product manifold of dimension $m \geq 3$ with a compatible metric $g=g_{1} \oplus \cdots \oplus g_{l}$. We define $\operatorname{Ric}_{i}^{g} \in \Gamma\left(\operatorname{Sym}^{2} T^{*} M\right)$ and $R_{i}^{g} \in C^{\infty}(M)$ by

$$
\begin{aligned}
\operatorname{Ric}_{i}^{g}(X, Y) & =\operatorname{Ric}^{g}\left(\mathcal{P}_{i} X, \mathcal{P}_{i} Y\right), \\
R_{i}^{g} & =\left\langle\operatorname{Ric}^{g}, g_{i}\right\rangle=\operatorname{tr}^{g} \operatorname{Ric}_{i}^{g}
\end{aligned}
$$

for all $X, Y \in \Gamma(T M)$, where $\mathcal{P}_{i}$ denotes the orthogonal projection onto the subbundle $E_{i}$. Note that

$$
R^{g}=R_{1}^{g}+\cdots+R_{l}^{g}
$$

always holds while $\operatorname{Ric}^{g}=\operatorname{Ric}_{1}^{g}+\cdots+\operatorname{Ric}_{l}^{g}$ if and only if $\operatorname{Ric}^{g}\left(\mathcal{P}_{i} X, \mathcal{P}_{j} Y\right)=0$ for all $X, Y \in \Gamma(T M)$ whenever $i \neq j$.

Proposition 2.3 Let $g=g_{1} \oplus \cdots \oplus g_{l} \in \mathrm{Met}_{\perp}$ be a compatible metric on a closed connected almost product manifold $M$.

(1) $g$ is critical with respect to the functional $E$ restricted to $\llbracket g \rrbracket$ if and only if there exists a real number $c$ independent of $i$ such that $R_{i}^{g} / m_{i}=c$ for all $i \in\{1, \ldots, l\}$, where $m_{i}=\operatorname{rank}\left(E_{i}\right)$.

(2) $g$ is critical with respect to the functional E restricted to $\mathrm{Met}_{\perp}$ if and only if there exists a constant $c$ independent of $i$ such that $\operatorname{Ric}_{i}^{g}=c g_{i}$ for all $i \in\{1, \ldots, l\}$.

Proof First we observe that any metric $g$ that is critical for $E$ restricted to $\llbracket g \rrbracket$ or Met $\perp$ also has to be critical for $E$ restricted to $[g]$ as $[g] \subset \llbracket g \rrbracket \subset$ Met $_{\perp}$. Thus, any critical value $g$ for $E$ restricted to $\llbracket g \rrbracket$ or $\mathrm{Met}_{\perp}$ has constant scalar curvature.

To show (1) we first assume that $g$ is critical for $\left.E\right|_{\llbracket g \rrbracket}$. Since a section $h \in \Gamma\left(\operatorname{Sym}^{2} T^{*} M\right)$ is tangent to $\llbracket g \rrbracket$ if and only if $h=\varphi_{1} g_{1} \oplus \cdots \oplus \varphi_{l} g_{l}$ for some $\varphi_{i} \in C^{\infty}(M), i \in\{1, \ldots, l\}$, it follows that

$$
0=\left.\frac{d}{d t}\right|_{t=0} E\left(g+t \varphi_{i} g_{i}\right)
$$




$$
=-\int_{M}\left\langle\varphi_{i} g_{i}, \operatorname{Ric}^{g}-\left(R^{g} / m\right) g\right\rangle d \mu^{g}=-\int_{M} \varphi_{i}\left(R_{i}^{g}-\left(R^{g} / m\right) m_{i}\right) d \mu^{g}
$$

for all $\varphi_{i} \in C^{\infty}(M)$ and $1 \leq i \leq l$ by (2.1). Thus, $R_{i}^{g} / m_{i}=R^{g} / m$. For the other direction we assume $R_{1}^{g} / m_{1}=\cdots=R_{l}^{g} / m_{l}=c$. Then $R_{i}^{g} / m_{i}=R^{g} / m$ necessarily holds by (2.6), and

$$
\begin{aligned}
\left.\frac{d}{d t}\right|_{t=0} E\left(g+t\left(\varphi_{1} g_{1} \oplus \cdots \oplus \varphi_{l} g_{l}\right)\right) & =-\int_{M}\left\langle\varphi_{1} g_{1} \oplus \cdots \oplus \varphi_{l} g_{l}, \operatorname{Ric}^{g}-\left(R^{g} / m\right) g\right\rangle d \mu^{g} \\
& =-\sum_{i=1}^{l} \int_{M} m_{i} \varphi_{i}\left(R_{i}^{g} / m_{i}-R^{g} / m\right) d \mu^{g}=0
\end{aligned}
$$

for all $\varphi_{1}, \ldots, \varphi_{l} \in C^{\infty}(M)$ by (2.1). Hence, $g$ is critical for $\left.E\right|_{\llbracket g \rrbracket}$.

The second statement is shown analogously. Observe that a section $h \in \Gamma\left(\operatorname{Sym}^{2} T^{*} M\right)$ is tangent to Met $\perp$ if and only if $h$ can be written as a sum $h=h_{1} \oplus \cdots \oplus h_{l}$ of sections $h_{i} \in \Gamma\left(\operatorname{Sym}^{2} E_{i}^{*}\right)$. First, we assume that $g$ is critical for $\left.E\right|_{\operatorname{Met}_{\perp}}$. Then,

$$
0=\left.\frac{d}{d t}\right|_{t=0} E\left(g+t h_{i}\right)=-\int_{M}\left\langle h_{i}, \operatorname{Ric}^{g}-\left(R^{g} / m\right) g\right\rangle d \mu^{g}
$$

for all $h_{i} \in \Gamma\left(\operatorname{Sym}^{2} E_{i}^{*}\right)$ and each $1 \leq i \leq l$ by $(2.1)$, whence $0=\operatorname{Ric}_{i}^{g}-\left(R^{g} / m\right) g_{i}$.

Conversely, assuming $\operatorname{Ric}_{i}^{g}=c g_{i}$ for all $i \in\{1, \ldots, l\}$ implies $c=R_{1}^{g} / m_{1}=\cdots=$ $R_{l}^{g} / m_{l}$. Thus, $c=R^{g} / m$ by (2.6) and therefore

$$
\begin{aligned}
\left.\frac{d}{d t}\right|_{t=0} E\left(g+t\left(h_{1} \oplus \cdots \oplus h_{l}\right)\right) & =-\int_{M}\left\langle h_{1} \oplus \cdots \oplus h_{l}, \operatorname{Ric}^{g}-\left(R^{g} / m\right) g\right\rangle d \mu^{g} \\
& =-\sum_{i=1}^{l} \int_{M}\left\langle h_{i}, \operatorname{Ric}_{i}^{g}-\left(R^{g} / m\right) g_{i}\right\rangle d \mu^{g}=0
\end{aligned}
$$

for all $h_{i} \in \Gamma\left(\operatorname{Sym}^{2} E_{i}^{*}\right), i \in\{1, \ldots, l\}$. This shows that $g$ is critical for $\left.E\right|_{\text {Met }_{\perp}}$.

\section{The scalar curvature of a multiconformal class}

Let $M$ be a closed almost product manifold with the splitting $T M=\bigoplus_{i=1}^{l} E_{i}$ and let $g=$ $g_{1} \oplus \cdots \oplus g_{l}$ be a compatible metric. Further, for each $i \in\{1, \ldots l\}$ we set $m_{i}:=\operatorname{rank}\left(E_{i}\right)$ and denote by $\mathcal{P}_{i}$ the corresponding orthogonal projection onto $E_{i}$. Now, for each $f \in C^{\infty}(M)$ we define

$$
\begin{aligned}
d_{i} f(X) & :=d f\left(\mathcal{P}_{i} X\right), \\
\operatorname{grad}_{i}^{g} f & :=\mathcal{P}_{i} \operatorname{grad}^{g}(f), \\
\operatorname{Hess}_{i}^{g} f(X, Y) & :=\operatorname{Hess}^{g} f\left(\mathcal{P}_{i}(X), \mathcal{P}_{i} Y\right), \\
\Delta_{i}^{g} f & :=\operatorname{tr}^{g} \operatorname{Hess}_{i}^{g} f .
\end{aligned}
$$

As we are interested in the behavior of the scalar curvature of the metrics in a fixed multiconformal class $\llbracket g \rrbracket$ we calculate the scalar curvature of a multiconformal change $\tilde{g}=f_{1}^{2} g_{1} \oplus \cdots \oplus f_{l}^{2} g_{l}$ in terms of the scalar curvature of $g_{i}$ and the multiconformal factors $f_{1}, \ldots, f_{l}$. Since the involved calculations are lengthy but straight-forward we moved them to the "Appendix A" and only state the final result below. 
Theorem 3.1 Let $M$ be an almost product manifold with the decomposition $T M=\bigoplus_{i=1}^{l} E_{i}$ and an compatible metric $g$. The scalar curvature $R^{\tilde{g}}$ of a multiconformal change $\tilde{g}=$ $f_{1}^{2} g_{1} \oplus \cdots \oplus f_{l}^{2} g_{l}$ is given by

$$
R^{\tilde{g}}=\sum_{i=1}^{l} \frac{R_{i}^{g}}{f_{i}^{2}}+\sum_{i=1}^{l} \frac{\rho_{i}}{f_{i}^{2}}
$$

where $\rho_{i}$ is defined by

$$
\begin{aligned}
\rho_{i}= & \rho_{i}^{g}\left(f_{1}, \ldots, f_{l}\right) \\
= & -2\left(m_{i}-1\right) \frac{\Delta_{i}^{g} f_{i}}{f_{i}}-2 \sum_{j \neq i} m_{j} \frac{\Delta_{i}^{g} f_{j}}{f_{j}} \\
& -\left(m_{i}-1\right)\left(m_{i}-4\right) \frac{\left|\operatorname{grad}_{i}^{g} f_{i}\right|_{g}^{2}}{f_{i}^{2}}-2\left(m_{i}-2\right) \sum_{j \neq i} m_{j} \frac{g\left(\operatorname{grad}_{i}^{g} f_{i}, \operatorname{grad}_{i}^{g} f_{j}\right)}{f_{i} f_{j}} \\
& -\sum_{j \neq i} m_{j}\left(m_{j}-1\right) \frac{\left|\operatorname{grad}_{i}^{g} f_{j}\right|_{g}^{2}}{f_{j}^{2}}-\sum_{j \neq i, k \neq i, j \neq k} m_{j} m_{k} \frac{g\left(\operatorname{grad}_{i}^{g} f_{j}, \operatorname{grad}_{i}^{g} f_{k}\right)}{f_{j} f_{k}},
\end{aligned}
$$

where $m_{i}=\operatorname{rank}\left(E_{i}\right)$ for each $1 \leq i \leq l$.

Setting $f_{1}=\ldots=f_{l}=: f$ in the above theorem, yields the well-known formula

$$
R^{\tilde{g}}=\frac{1}{f^{2}}\left(R^{g}-2(m-1) \frac{\Delta^{g} f}{f}-(m-1)(m-4) \frac{|d f|^{2}}{f^{2}}\right)
$$

for the scalar curvature of the conformally defomed metric $\tilde{g}=f^{2} g$ and in the special case, where $f_{1} \equiv 1$ and $f_{i} \in C^{\infty}\left(M_{i}\right)$ for all $i>2$ yields the scalar curvature formula for the multiply warped product metric $g_{1} \oplus f_{2}^{2} g_{2} \oplus \cdots \oplus f_{l}^{2} g_{l}$, compare [13, Proposition 2.6].

Remark 3.2 When $g$ is a direct product metric, the multiconformally related metric $\tilde{g}$ is also called a twisted product. ${ }^{1}$ If in addition $l=2$, it is more common to say that $\tilde{g}$ is biconformal ${ }^{2}$ to $g$. Moreover, for a direct product metric $g$, a formula without proof for the curvature tensor of $\tilde{g}$ can be found in Meumertzheim-Reckziegel-Schaaf [28, Proposition 1]. In "Appendix A" we present here the detailed computation, which works for an arbitrary compatible metric.

Theorem 3.1 states a pointwise formula for the scalar curvature of a multiconformal change. In the next step, we derive an integral formulas based on the above theorem which will be used to prove the theorems from the introduction. However, as we want to use partial integration, we now assume that $(M, g)=\left(M_{1}, g_{1}\right) \times \cdots \times\left(M_{l}, g_{l}\right)$ is a direct product of closed and connected Riemannian manifolds $\left(M_{1}, g_{1}\right), \ldots,\left(M_{l}, g_{l}\right)$, i.e. $g$ is the induced product metric.

Proposition 3.3 Let $(M, g)=\left(M_{1}, g_{1}\right) \times \cdots \times\left(M_{l}, g_{l}\right)$ is the direct product of closed and connected Riemannian manifolds $\left(M_{1}, g_{1}\right), \ldots,\left(M_{l}, g_{l}\right)$ and let $\tilde{g}=f_{1}^{2} g_{1} \oplus \cdots \oplus f_{l}^{2} g_{l}$.

\footnotetext{
1 cf. Koike [26, p. 3], Meumertzheim-Reckziegel-Schaaf [28, Definition 2].

2 cf. Mo [29, p. 15], Slobodeanu [42,43], Ou [32, Lemma 2.1], Danielo [11, Définition 2.1], Rovenski-Zelenko [36, p. 504].
} 
Then, for each $\left(q_{1}, \ldots, q_{l}\right) \in \mathbb{R}^{l}$ we have the integral identity

$$
\begin{aligned}
& \int_{M}\left(R^{\tilde{g}}-\sum_{i=1}^{l} \frac{R_{i}^{g}}{f_{i}^{2}}\right) f_{1}^{q_{1}} \cdots f_{l}^{q_{l}} d \mu^{g} \\
& =\sum_{i=1}^{l} \int_{M}\left(\sum_{j, k=1}^{l} b_{j k}^{i} \frac{\left\langle\operatorname{grad}_{i}^{g} f_{j}, \operatorname{grad}_{i}^{g} f_{k}\right\rangle}{f_{j} f_{k}}\right) \frac{f_{1}^{q_{1}} \cdots f_{l}^{q_{l}}}{f_{i}^{2}} d \mu^{g},
\end{aligned}
$$

where $B^{i}=\left(b_{j k}^{i}\right)_{1 \leq j, k \leq l}$ are symmetric matrices given by

$$
\begin{aligned}
& B^{i}=-\left(m_{j} m_{k}-m_{j} q_{k}-m_{k} q_{j}\right)_{j k}
\end{aligned}
$$

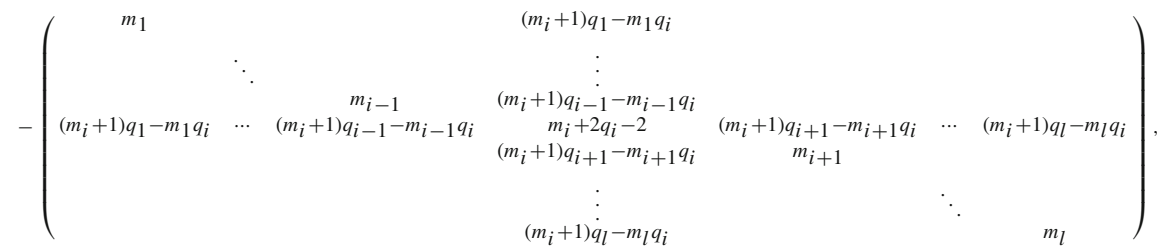

where $m_{i}=\operatorname{dim}\left(M_{i}\right)$

Proof Let $\left(q_{1}, \ldots, q_{l}\right) \in \mathbb{R}^{l}$ and consider the difference

$$
\int_{M}\left(R^{\tilde{g}}-\sum_{i=1}^{l} \frac{R_{i}^{g}}{f_{i}^{2}}\right) f_{1}^{q_{1}} \cdots f_{l}^{q_{l}} d \mu^{g} .
$$

It follows directly from Theorem 3.1 that this difference is equivalent to

$$
\begin{aligned}
& \int_{M} \frac{\rho_{i}}{f_{i}^{2}} f_{1}^{q_{1}} \cdots f_{l}^{q_{l}} d \mu^{g} \\
& =-2\left(m_{i}-1\right) \int_{M} \frac{\Delta_{i}^{g} f_{i}}{f_{i}^{3}} f_{1}^{q_{1}} \cdots f_{l}^{q_{l}} d \mu^{g}-2 \sum_{j \neq i} m_{j} \int_{M} \frac{\Delta_{i}^{g} f_{j}}{f_{i}^{2} f_{j}} f_{1}^{q_{1}} \cdots f_{l}^{q_{l}} d \mu^{g} \\
& \quad-\left(m_{i}-1\right)\left(m_{i}-4\right) \int_{M} \frac{\left|\operatorname{grad}_{i}^{g} f_{i}\right|_{g}^{2}}{f_{i}^{4}} f_{1}^{q_{1}} \cdots f_{l}^{q_{l}} d \mu^{g} \\
& \quad-2\left(m_{i}-2\right) \sum_{j \neq i} m_{j} \int_{M} \frac{\left\langle\operatorname{grad}_{i}^{g} f_{i}, \operatorname{grad}_{i}^{g} f_{j}\right\rangle}{f_{i}^{3} f_{j}} f_{1}^{q_{1}} \cdots f_{l}^{q_{l}} d \mu^{g} \\
& \quad-\sum_{j \neq i} m_{j}\left(m_{j}-1\right) \int_{M} \frac{\left|\operatorname{grad}_{i}^{g} f_{j}\right|_{g}^{2}}{f_{i}^{2} f_{j}^{2}} f_{1}^{q_{1}} \cdots f_{l}^{q_{l}} d \mu^{g} \\
& \quad-\sum_{j \neq i, k \neq i, j \neq k} m_{j} m_{k} \int_{M} \frac{\left\langle\operatorname{grad}_{i}^{g} f_{j}, \operatorname{grad}_{i}^{g} f_{k}\right\rangle}{f_{i}^{2} f_{j} f_{k}} f_{1}^{q_{1}} \cdots f_{l}^{q_{l}} d \mu^{g} .
\end{aligned}
$$


Using partial integration along each $M_{i}$ separately, we can get rid of the second derivatives. This leads to the identities

$$
\begin{aligned}
& -2\left(m_{i}-1\right) \int_{M} \frac{\Delta_{i}^{g} f_{i}}{f_{i}^{3}} f_{1}^{q_{1}} \cdots f_{l}^{q_{l}} d \mu^{g} \\
& =2\left(m_{i}-1\right)\left(q_{i}-3\right) \int_{M} \frac{\left|\operatorname{grad}_{i}^{g} f_{i}\right|^{2}}{f_{i}^{4}} f_{1}^{q_{1}} \cdots f_{l}^{q_{l}} d \mu^{g} \\
& \quad+2\left(m_{i}-1\right) \sum_{j \neq i} q_{j} \int_{M} \frac{\left\langle\operatorname{grad}_{i}^{g} f_{i}, \operatorname{grad}_{i}^{g} f_{j}\right\rangle}{f_{i}^{3} f_{j}} f_{1}^{q_{1}} \cdots f_{l}^{q_{l}} d \mu^{g}
\end{aligned}
$$

and

$$
\begin{aligned}
& -2 \sum_{j \neq i} m_{j} \int_{M} \frac{\Delta_{i}^{g} f_{j}}{f_{i}^{2} f_{j}} f_{1}^{q_{1}} \cdots f_{l}^{q_{l}} d \mu^{g} \\
& =2\left(q_{i}-2\right) \sum_{j \neq i} m_{j} \int_{M} \frac{\left\langle\operatorname{grad}_{i}^{g} f_{i}, \operatorname{grad}_{i}^{g} f_{j}\right\rangle}{f_{i}^{3} f_{j}} f_{1}^{q_{1}} \cdots f_{l}^{q_{l}} d \mu^{g} \\
& \quad+2 \sum_{j \neq i} m_{j}\left(q_{j}-1\right) \int_{M} \frac{\left|\operatorname{grad}_{i}^{g} f_{j}\right|^{2}}{f_{i}^{2} f_{j}^{2}} f_{1}^{q_{1}} \cdots f_{l}^{q_{l}} d \mu^{g} \\
& \quad+2 \sum_{j \neq i, k \neq i, j \neq k} m_{j} q_{k} \int_{M} \frac{\left\langle\operatorname{grad}_{i}^{g} f_{j}, \operatorname{grad}_{i}^{g} f_{k}\right\rangle}{f_{i}^{2} f_{j} f_{k}} f_{1}^{q_{1}} \cdots f_{l}^{q_{l}} d \mu^{g} .
\end{aligned}
$$

Inserting (3.5) and (3.6) into (3.4) the claim follows from

$$
\begin{aligned}
\int_{M} & \frac{\rho_{i}}{f_{i}^{2}} f_{1}^{q_{1}} \cdots f_{l}^{q_{l}} d \mu^{g} \\
= & \left(m_{i}-1\right)\left(2 q_{i}-m_{i}-2\right) \int_{M} \frac{\left|\operatorname{grad}_{i}^{g} f_{i}\right|^{2}}{f_{i}^{4}} f_{1}^{q_{1}} \cdots f_{l}^{q_{l}} d \mu^{g} \\
& +\sum_{j \neq i} m_{j}\left(2 q_{j}-m_{j}-1\right) \int_{M} \frac{\left|\operatorname{grad}_{i}^{g} f_{j}\right|^{2}}{f_{i}^{2} f_{j}^{2}} f_{1}^{q_{1}} \cdots f_{l}^{q_{l}} d \mu^{g} \\
& +2 \sum_{j \neq i}\left(m_{i} q_{j}+q_{i} m_{j}-m_{i} m_{j}-q_{j}\right) \int_{M} \frac{\left\langle\operatorname{grad}_{i}^{g} f_{i}, \operatorname{grad}_{i}^{g} f_{j}\right\rangle}{f_{i}^{3} f_{j}} f_{1}^{q_{1}} \cdots f_{l}^{q_{l}} d \mu^{g} \\
& +\sum_{j \neq i, k \neq i, j \neq k}\left(m_{j} q_{k}+q_{j} m_{k}-m_{j} m_{k}\right) \int_{M} \frac{\left\langle\operatorname{grad}_{i}^{g} f_{j}, \operatorname{grad}_{i}^{g} f_{k}\right\rangle}{f_{i}^{2} f_{j} f_{k}} f_{1}^{q_{1}} \cdots f_{l}^{q_{l}} d \mu^{g} \\
= & \sum_{i=1}^{l} \int_{M}\left(\sum_{j, k=1}^{l} b_{j k}^{i} \frac{\left\langle\operatorname{grad}_{i}^{g} f_{j}, \operatorname{grad}_{i}^{g} f_{k}\right\rangle}{f_{j} f_{k}}\right) \frac{f_{1}^{q_{1}} \cdots f_{l}^{q_{l}}}{f_{i}^{2}} d \mu_{g} .
\end{aligned}
$$

In the view of Theorem 1.1 we want to be able to determine the sign of the difference $R^{\tilde{g}}-\sum_{i} \frac{R^{g}}{f_{i}^{2}}$. There, the following change of variables will come into use.

Lemma 3.4 Let $(M, g)$ be a Riemannian manifold, $E$ a vector subbundle of $T M$, et $\mathcal{P}$ be the orthogonal projection, and let $f_{1}, \ldots, f_{l} \in C^{\infty}(M)$ be a family of positive functions. For 
each choice of a vector $a=\left(a_{1}, \ldots, a_{l}\right) \in \mathbb{R}^{l}$, a symmetric matrix $B=\left(b_{j k}\right)_{1 \leq j, k \leq l}$, and an orthogonal matrix $P=\left(p_{j, k}\right)_{1 \leq j, k \leq l}$,

$$
\begin{aligned}
& \sum_{j=1}^{l} a_{j} \frac{\Delta_{E}^{g} f_{j}}{f_{j}}+\sum_{j, k=1}^{l} b_{j k} \frac{\left\langle d_{E} f_{j}, d_{E} f_{k}\right\rangle}{f_{j} f_{k}} \\
& \quad=\sum_{\alpha, i=1}^{l} p_{\alpha i} a_{i} \Delta_{E}^{g} \psi_{\alpha}+\sum_{\alpha, \beta, j, k=1}^{l} p_{\alpha i} p_{\beta k}\left(\delta_{j k} a_{j}+b_{j k}\right)\left\langle d_{E} \psi_{\alpha}, d_{E} \psi_{\beta}\right\rangle,
\end{aligned}
$$

where $\psi_{\alpha}=\sum_{j} p_{\alpha j} \log \left(f_{j}\right)$ for all $1 \leq \alpha \leq l$. Here, the operators $\Delta_{E}$ and $d_{E}$ are defined as in (3.1).

Proof We consider the section

$$
\sum_{j=1}^{l} a_{j} \frac{\operatorname{Hess}_{E}^{g} f_{j}}{f_{j}}+\sum_{j, k=1}^{l} b_{j k} \frac{d_{E} f_{j} \otimes d_{E} f_{k}}{f_{i} f_{k}}
$$

of Sym ${ }^{2} T^{*} M$, where $a=\left(a_{1}, \ldots, a_{l}\right) \in \mathbb{R}^{l}$ and $B=\left(b_{j} k\right)_{1 \leq j, k \leq l}$ is a symmetric matrix. Here, the operators $d_{E}$ and $\operatorname{Hess}_{E}^{g}$ are defined analogously to (3.1), i.e.

$$
\begin{aligned}
d_{E} f(X) & =d f(\mathcal{P} X), \\
\operatorname{Hess}_{E}^{g} f(X, Y) & =\operatorname{Hess}^{g} f(\mathcal{P} X, Y),
\end{aligned}
$$

for any $X, Y \in T M$ and any smooth function $f$. Setting $u_{j}:=\log f_{j}$ for each $1 \leq j \leq l$ we obtain

$$
\begin{aligned}
& \sum_{j=1}^{l} a_{j} \frac{\operatorname{Hess}_{E}^{g} f_{j}}{f_{j}}+\sum_{j, k=1}^{l} b_{j k} \frac{d_{E} f_{j} \otimes d_{E} f_{k}}{f_{j} f_{k}} \\
& \quad=\sum_{j=1}^{l} a_{j} \operatorname{Hess}_{E}^{g} u_{j}+\sum_{j=1}^{l}\left(a_{j}+b_{j j}\right) d_{E} u_{j} \otimes d_{E} u_{j}+\sum_{j \neq k} b_{j k} d_{E} u_{j} \otimes d_{E} u_{k} .
\end{aligned}
$$

Next, we use the orthogonal matrix $P=\left(p_{j k}\right)_{1 \leq j, k \leq l}$ and define $\psi_{\alpha}:=\sum_{j=1}^{l} p_{\alpha j} u_{j}$. Since $P$ is an orthogonal matrix, it follows that $u_{j}=\sum_{\alpha=1}^{l} p_{\alpha j} p_{\alpha j} \psi_{\alpha}$. Inserting this relation into the above equation we obtain

$$
\begin{aligned}
& \sum_{j=1}^{l} a_{j} \operatorname{Hess}_{E}^{g} u_{j}+\sum_{j=1}^{l}\left(a_{j}+b_{j j}\right) d_{E} u_{j} \otimes d_{E} u_{j}+\sum_{j \neq k} b_{j k} d_{E} u_{j} \otimes d_{E} u_{k} \\
& =\sum_{j=1}^{l} a_{j} \operatorname{Hess}_{E}^{g} u_{j}+\sum_{j, k=1}^{l}(A+B)_{j k} d_{E} u_{j} \otimes d_{E} u_{k} \\
& =\sum_{j, \alpha=1}^{l} a_{j} p_{\alpha j} \operatorname{Hess}_{E}^{g} \psi_{\alpha}+\sum_{j, k, \alpha, \beta=1}^{l}(A+B)_{j k} p_{\alpha j} p_{\beta k} d_{E} \psi_{\alpha} \otimes d_{E} \psi_{\beta}
\end{aligned}
$$

Taking the trace of both sides, the claim follows.

Corollary 3.5 If in the above lemma, the matrix $B$ is positive (resp. negative) definite, then $\sum_{j, k=1}^{l} b_{j k} \frac{\left\langle d_{E} f_{j}, d_{E} f_{k}\right\rangle}{f_{j} f_{k}}$ is nonpositive (resp. nonnegative) if and only if $d_{E} f_{1}=\cdots=d_{E} f_{l}=$ 0 . 
Proof We apply Lemma 3.4 with $a=0$ and $P=$ Id. Then,

$$
\sum_{j, k=1}^{l} b_{j k} \frac{\left\langle d_{E} f_{j}, d_{E} f_{k}\right\rangle}{f_{j} f_{k}}=\sum_{j, k=1} b_{j k}\left\langle d_{E}\left(\log f_{j}\right), d_{E}\left(\log f_{k}\right)\right\rangle .
$$

By assumption $B$ is positive definite, hence the right hand side is nonpositive if and only if $d_{E}\left(\log f_{j}\right)=0$ for all $1 \leq j \leq l$. Thus, if and only if $d_{E} f_{j}=0$ for all $j=1, \ldots, l$. If $B$ is negative definite, the same conclusion holds if the left hand side of (3.7) is nonnegative.

\section{The sign of a multiconformal class}

Let $(M, g)=\left(M_{1}, g_{1}\right) \times \cdots \times\left(M_{l}, g_{l}\right)$ be a direct product of closed connected Riemannian manifolds and recall the invariant

$$
\sigma(M, \llbracket g \rrbracket)=\sup _{[\tilde{g}] \in \llbracket g \rrbracket} \mu(M,[\tilde{g}])
$$

defined in Sect. 2. There we also showed that $\sigma(M, \llbracket g \rrbracket)$ is positive if and only if there is a metric $\tilde{g} \in \llbracket g \rrbracket$ of positive scalar curvature. But can be even more precise. Below we prove Theorem 1.1 which implies that $\sigma(M, \llbracket g \rrbracket)$ is positive if and only if one of the factors admits a positive scalar curvature metric.

Theorem 1.1 Let $(M, g)=\left(M_{1}, g_{1}\right) \times \cdots \times\left(M_{l}, g_{l}\right)$ be a direct product of closed connected Riemannian manifolds such that $\operatorname{dim}\left(M_{i}\right) \geq 2$ for all $1 \leq i \leq l$. Then the following trichotomy holds.

(1) The multiconformal class $\llbracket g \rrbracket$ contains a metric of positive scalar curvature if and only if there exists $i \in\{1, \ldots, l\}$ such that $\mu\left(M_{i},\left[g_{i}\right]\right)>0$.

(2) The multiconformal class $\llbracket g \rrbracket$ does not contain a metric of positive scalar curvature and there exists a scalar flat metric of $\llbracket g \rrbracket$ if and only if $\mu\left(M_{i},\left[g_{i}\right]\right)=0$ for every $i \in\{1, \ldots, l\}$. In this case, if $\tilde{g} \in \llbracket g \rrbracket$ has nonnegative scalar curvature, then $\tilde{g}$ is necessarily scalar flat and direct product.

(3) The multiconformal class $\llbracket g \rrbracket$ does not contain a metric of nonnegative scalar curvature if and only if for every $i \in\{1, \ldots, l\}, \mu\left(M_{i},\left[g_{i}\right]\right) \leq 0$ and there exists an $i \in\{1, \ldots, l\}$ such that $\mu\left(M_{i},\left[g_{i}\right]<0\right)$.

Proof First we observe that the statements of the theorem only deal with the sign of the conformal Yamabe invariant of the factors. By the resolution of the Yamabe problem, we know that each closed Riemannian manifold $(M, g)$ can be conformally deformed into a Riemannian manifold $(M, \tilde{g})$ of constant scalar curvature and volume one. In this case, the scalar curvature is equal to $\mu(M,[g])$. Thus, we can assume without loss of generality that $g_{i}$ is a constant scalar curvature metric and $\operatorname{Vol}\left(M_{i}, g_{i}\right)=1$ for all $1 \leq i \leq l$.

Before going into each case separately, we make the following general observations:

For any $\tilde{g}=f_{1}^{2} g_{1} \oplus \cdots \oplus f_{l}^{2} g_{l} \in \llbracket g \rrbracket$ we apply Proposition 3.3 with $q_{1}=\cdots=q_{l}=0$ and obtain

$$
\int_{M}\left(R^{\tilde{g}}-\sum_{i=1}^{l} \frac{R_{i}^{g}}{f_{i}^{2}}\right) d \mu^{g} \leq \sum_{i=1}^{l} \int_{M}\left(\sum_{j, k=1}^{l} b_{j k}^{i} \frac{\left\langle\operatorname{grad}_{i}^{g} f_{j}, \operatorname{grad}_{i}^{g} f_{k}\right\rangle}{f_{j} f_{k}}\right) \frac{1}{f_{i}^{2}} d \mu^{g} .
$$


Here, $\left(b_{j k}^{i}\right)_{j k}$ are the entries of the symmetric matrix

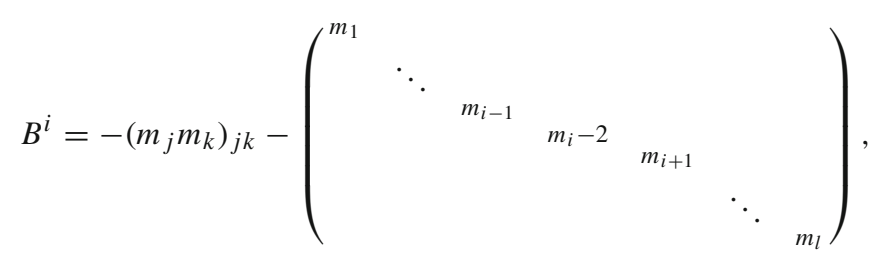

where $m_{i}=\operatorname{dim}\left(M_{i}\right)$ for $1 \leq i \leq l$.

We claim that the right hand side of (4.1) is nonpositive. By Corollary 3.5 this is the case if all the matrices $B^{i}$ are negative definite for any $1 \leq i \leq l$. To see this, let $x \in \mathbb{R}^{l}$. Then

$$
\left\langle x, B^{i} x\right\rangle=-\left(\sum_{j=1}^{l} x_{j}\right)^{2}-\sum_{j=1}^{l}\left(m_{j}-2 \delta_{i j}\right) x_{j}^{2} .
$$

It is not hard to see that $\left(\sum_{j} m_{j} x_{j}\right)^{2} \leq 0$ and equality holds if and only if $\left(x_{1}, \ldots, x_{l}\right) \perp$ $\left(m_{1}, \ldots, m_{l}\right)$. Analogously, $\left.\sum_{j}\left(m_{j}-2 \delta_{i j}\right) x_{j}^{2}\right) \leq 0$ with equality if and only if $m_{i}=2$ and $x=\left(0, \ldots, 0, x_{i}, 0, \ldots, 0\right)$. Since these two conditions can not be satisfies simultaneously, it follows that $B^{i}$ is negative definite. Hence, we can apply Corollary 3.5 to (4.1) and conclude that

$$
\int_{M}\left(R^{\tilde{g}}-\sum_{i=1}^{l} \frac{R_{i}^{g}}{f_{i}^{2}}\right) d \mu^{g} \leq 0
$$

where equality holds if and only if $f_{1}, \ldots, f_{l}$ are all constant.

(1) If $\llbracket g \rrbracket$ contains a metric $\tilde{g}$ of positive scalar curvature, then it follows from (4.2) that

$$
0<R^{\tilde{g}} \leq \sum_{i=1}^{l} \int_{M} \frac{R_{i}^{g}}{f_{i}^{2}} d \mu^{g} .
$$

As $f_{1}, \ldots, f_{l}$ are positive functions, there has to be at least one $i \in\{1, \ldots, l\}$ such that $R^{g}>0$, which is equivalent to $\mu\left(M_{i},\left[g_{i}\right]\right)>0$ by our choice of the metric $g_{i}$. On the other hand, if $\mu\left(M_{i},\left[g_{i}\right]\right)>0$ for some $i \in\{1, \ldots, l\}$, we can choose a positive scalar curvature metric on $M_{i}$. Then an appropriate scaling of the single factors leads to a positive scalar curvature metric.

(2) If $\llbracket g \rrbracket$ does not contain a metric of positive scalar curvature but a scalar flat metric $\tilde{g}$, then

$$
0=R^{\tilde{g}} \leq \sum_{i=1}^{l} R^{g_{i}} \int_{M} f_{i}^{-2} d \mu^{g}
$$

by (4.2). Moreover, $\mu\left(M_{i},\left[g_{i}\right]\right) \leq 0$ for all $1 \leq i \leq l$ as otherwise there would be a metric of positive scalar curvature in $\llbracket g \rrbracket$. Thus, it follows that the above inequality is satisfied if and only if $R^{g_{i}}=0$, i.e. $\mu\left(M_{i},\left[g_{i}\right]\right)=0$ for all $1 \leq i \leq l$. As in this case the above inequality is in fact an equality the functions $f_{1}, \ldots, f_{l}$ have to be constant. In particular, the scalar flat metric $\tilde{g}$ is a product metric.

Lastly, (3) is now an immediate consequence from (1) and (2). 
Remark 4.1 Theorem 1.1 has the technical assumption that no factor $M_{i}$ can be diffeomorphic to $S^{1}$. In the other extreme case where every factor $M_{i}$ is diffeomorphic to $S^{1}$, the enlargeability obstruction (Gromov-Lawson [21,22]) or the stable minimal hypersurface obstruction (Schoen-Yau [39-41]) show that $(M, g)=S^{1}(1) \times \cdots \times S^{1}(1)$ falls into case (2) of Theorem 1.1. It is interesting to ask whether our dimensional assumption $m_{1}, \ldots, m_{l} \geq 2$ can be removed.

As remarked in Sect. 2, $\sigma(M)>0(\operatorname{resp} . \sigma(M, \llbracket g \rrbracket)>0)$ if and only if $M($ resp. $\llbracket g \rrbracket)$ contains a metric of positive scalar curvature. However, it is well known that the KazdanWarner trichotomy (cf. [35, Theorem 0.1]) does not completely correspond to the sign of $\sigma(M)$. That is, if $M$ does not carry a positive scalar curvature metric but a scalar flat one, then $\sigma(M)=0$, but the converse does not hold in general.

A similar discrepancy holds for direct product Riemannian manifolds $(M, g)=$ $\left(M_{1}, g_{1}\right) \times \cdots \times\left(M_{l}, g_{l}\right)$ such that $\operatorname{dim}\left(M_{i}\right) \geq 2$ for all $1 \leq i \leq l$. If $\sigma(M, \llbracket g \rrbracket)=0$ then it is a direct consequence of Theorem 1.1 that $\mu\left(M_{i},\left[g_{i}\right]\right) \leq 0$ for all $1 \leq i \leq l$ and the maximum is attained if and only if $\mu\left(M_{i},\left[g_{i}\right]\right)=0$ for all $1 \leq i \leq l$. However, there are many cases, where the maximum is not attained. For example, if $\mu\left(M_{1},\left[g_{1}\right]\right)=0$, $\mu\left(M_{2},\left[g_{2}\right]\right)<0$ and $\mu\left(M_{i},\left[g_{i}\right]\right) \leq 0$ for all $3 \leq i \leq l$ then a simple scaling argument shows that $\sigma(M, \llbracket g \rrbracket)=0$ but there is no maximizing metric in $\llbracket g \rrbracket$. It is an interesting question, whether $\mu\left(M_{i},\left[g_{i}\right]\right)<0$ for all $1 \leq i \leq l$ implies $\sigma(M, \llbracket g \rrbracket)<0$.

\section{The infimum of the conformal Yamabe invariants}

In Sect. 4 we discussed the supremum of the conformal Yamabe invariants within a multiconformal class. While the sign of the invariant $\sigma(M, \llbracket g \rrbracket)$ of a direct product manifold $(M, g)=\left(M_{1}, g_{1}\right) \times \cdots \times\left(M_{l}, g_{l}\right)$ of closed connected Riemannian manifold is related to the sign of the conformal Yamabe invariants of its factors, see Theorem 1.1, the infinum is always $-\infty$.

Theorem 1.2 Let $(M, g)=\left(M_{1}, g_{1}\right) \times \cdots \times\left(M_{l}, g_{l}\right)$ be a direct product of closed connected Riemannian manifolds with $l \geq 2$ and $\operatorname{dim}(M) \geq 3$, then

$$
\inf _{[\tilde{g}] \subset \llbracket g \rrbracket} \mu(M,[\tilde{g}])=-\infty .
$$

Proof Without loss of generality we assume that $\operatorname{dim}\left(M_{1}\right) \geq 2$ (if necessary, consider the product manifold $M_{1} \times M_{2}$ as one factor). In the following, we construct a family of metrics $\tilde{g}_{\varepsilon}$ such that $\lim _{\varepsilon \rightarrow 0} \mu\left(M,\left[\tilde{g}_{\varepsilon}\right]\right)=-\infty$.

First, we fix a function $\varphi: M \rightarrow \mathbb{R}$ and consider the metric

$$
\tilde{g}=f_{1}^{2} g_{1} \oplus f_{2}^{2} g_{2} \oplus g_{3} \oplus \cdots \oplus g_{l},
$$

with $f_{i}=\exp \left(a_{i} \circ \varphi\right)$ with smooth functions $a_{i}: \mathbb{R} \rightarrow \mathbb{R}$ to be chosen. Next, we apply Proposition 3.3 to $\tilde{g}$ with $\left(q_{1}, \ldots, q_{l}\right)=\left(m_{1}, \ldots, m_{l}\right)$, where $m_{i}=\operatorname{dim}\left(M_{i}\right)$, and obtain 


$$
\begin{aligned}
& \int_{M}\left(R^{\tilde{g}}-\frac{R_{1}^{g}}{f_{1}^{2}}-\frac{R_{2}^{g}}{f_{2}^{2}}-\sum_{i=3}^{l} R_{i}^{g}\right) f_{1}^{m_{1}} f_{2}^{m_{2}} d \mu^{g} \\
& =\sum_{i=1}^{2} \int_{M}\left(\sum_{j, k=1}^{2} b_{j k}^{i} \frac{\left\langle\operatorname{grad}_{i}^{g} f_{j}, \operatorname{grad}_{i}^{g} f_{k}\right\rangle}{f_{j} f_{k}}\right) \frac{f_{1}^{m_{1}} f_{2}^{m_{2}}}{f_{i}^{2}} d \mu^{g} \\
& =\sum_{i=1}^{2} \int_{M}\left(\sum_{j, k=1} b_{j k}^{i}\left(a_{j}^{\prime} \circ \varphi\right)\left(a_{k}^{\prime} \circ \varphi\right)\left|\operatorname{grad}_{i} \varphi\right|^{2}\right) \frac{f_{1}^{m_{1}} f_{2}^{m_{2}}}{f_{i}^{2}} d \mu^{g},
\end{aligned}
$$

where

$$
\begin{aligned}
\left(b_{j k}^{1}\right)_{j k} & =\left(\begin{array}{cc}
\left(m_{1}-1\right)\left(m_{1}-2\right) & \left(m_{1}-1\right) m_{2} \\
\left(m_{1}-1\right) m_{2} & m_{2}\left(m_{2}-1\right)
\end{array}\right), \\
\left(b_{j k}^{2}\right)_{j k} & =\left(\begin{array}{lc}
m_{1}\left(m_{1}-1\right) & m_{1}\left(m_{2}-1\right) \\
m_{1}\left(m_{2}-1\right) & \left(m_{2}-1\right)\left(m_{2}-2\right)
\end{array}\right) .
\end{aligned}
$$

Now, we want to choose the functions $a_{1}, a_{2}: \mathbb{R} \rightarrow \mathbb{R}$ such that

$$
\int_{M}\left(\sum_{j, k=1} b_{j k}^{1}\left(a_{j}^{\prime} \circ \varphi\right)\left(a_{k}^{\prime} \circ \varphi\right)\left|\operatorname{grad}_{1} \varphi\right|^{2}\right) f_{1}^{m_{1}-2} f_{2}^{m_{2}} d \mu^{g}<-\int_{M} R_{1}^{g} f_{1}^{m_{1}-2} f_{2}^{m_{2}} d \mu^{g} .
$$

To do so, we set

$$
\begin{aligned}
& a_{1}(\theta)=\sin (\sqrt{\alpha} \theta), \\
& a_{2}(\theta)=-\beta \sin (\sqrt{\alpha} \theta) .
\end{aligned}
$$

for positive real numbers $\alpha, \beta$ to be specified later. Inserting this relation into the right hand side of (5.1) we obtain

$$
\begin{aligned}
& \int_{M}\left(\sum_{j, k=1} b_{j k}^{1}\left(a_{j}^{\prime} \circ \varphi\right)\left(a_{k}^{\prime} \circ \varphi\right)\left|\operatorname{grad}_{1} \varphi\right|^{2}\right) f_{1}^{m_{1}-2} f_{2}^{m_{2}} d \mu^{g} \\
& =\left(m_{1}-1\right)\left(m_{1}-2\right) \int_{M} \alpha \cos ^{2}(\varphi)\left|\operatorname{grad}_{1} \varphi\right|^{2} e^{\left(m_{1}-2-\beta m_{2}\right) \sin (\sqrt{\alpha} \varphi)} d \mu^{g} \\
& \quad-2\left(m_{1}-1\right) m_{2} \int_{M} \alpha \beta \cos ^{2}(\varphi)\left|\operatorname{grad}_{1} \varphi\right|^{2} e^{\left(m_{1}-2-\beta m_{2}\right) \sin (\sqrt{\alpha} \varphi)} d \mu^{g} \\
& \quad+m_{2}\left(m_{2}-1\right) \int_{M} \alpha \beta^{2} \cos ^{2}(\varphi)\left|\operatorname{grad}_{1} \varphi\right|^{2} e^{\left(m_{1}-2-\beta m_{2}\right) \sin (\sqrt{\alpha} \varphi)} d \mu^{g} \\
& =:-\alpha \gamma \int_{M} \cos ^{2}(\varphi)\left|\operatorname{grad}_{1} \varphi\right|^{2} e^{\left(m_{1}-2-\beta m_{2}\right) \sin (\sqrt{\alpha} \varphi)} d \mu^{g},
\end{aligned}
$$

where we set

$$
\gamma=-m_{2}\left(m_{2}-1\right) \beta^{2}+2\left(m_{1}-1\right) m_{2} \beta-\left(m_{1}-1\right)\left(m_{2}-2\right) .
$$


It is not hard to see, that we can always choose $\beta$ such that $\gamma$ is positive. To be more precise, if $m_{2}=1$, we simply take $\beta$ sufficiently large, and if $m_{2} \geq 2$, then we take $\beta$ slightly smaller than the larger root of the corresponding quadratic form. With this choice for $\beta$ we conclude,

$$
\begin{aligned}
& \int_{M}\left(\sum_{j, k=1} b_{j k}^{1}\left(a_{j}^{\prime} \circ \varphi\right)\left(a_{k}^{\prime} \circ \varphi\right)\left|\operatorname{grad}_{1} \varphi\right|^{2}\right) f_{1}^{m_{1}-2} f_{2}^{m_{2}} d \mu^{g} \\
& \quad=-\alpha \gamma \int_{M} \cos ^{2}(\varphi)\left|\operatorname{grad}_{1} \varphi\right|^{2} e^{\left(m_{1}-2-\beta m_{2}\right) \sin (\sqrt{\alpha} \varphi)} d \mu^{g} \\
& \leq-\alpha \gamma e^{-\left|m_{1}-2-\beta m_{2}\right|} \int_{M} \cos ^{2}(\varphi)\left|\operatorname{grad}_{1} \varphi\right|^{2} d \mu^{g} .
\end{aligned}
$$

Hence, for any fixed $\beta$ such that $\gamma>0$ we can choose $\alpha$ sufficiently large such that the inequality (5.1) is fulfilled. Fixing such a choice of $\alpha$ and $\beta$ finally defines our metric $\tilde{g}$.

For any $\varepsilon>0$ we define

$$
\tilde{g}_{\varepsilon}:=\varepsilon^{2} f_{1}^{2} g_{1} \oplus f_{2}^{2} g_{2} \oplus g_{3} \oplus \cdots \oplus g_{l},
$$

where $f_{1}, f_{2}: M \rightarrow \mathbb{R}_{+}$are the functions constructed above. It remains to show that $\lim _{\varepsilon \rightarrow 0} \mu\left(M,\left[\tilde{g}_{\varepsilon}\right]\right)=-\infty$. To see this, we apply the normalized Einstein-functional to $\tilde{g}_{\varepsilon}$ and use Proposition 3.3,

$$
\begin{aligned}
E\left(\tilde{g}_{\varepsilon}\right)= & \frac{\int_{M} R^{\tilde{g}_{\varepsilon}} d \mu^{\tilde{g}_{\varepsilon}}}{\operatorname{Vol}\left(M, \tilde{g}_{\varepsilon}\right)^{\frac{m-2}{m}}} \\
= & \varepsilon^{2\left(\frac{m_{1}}{m}-1\right)} \frac{\int_{M}\left(R_{1}^{g}+\sum_{j, k=1}^{2} b_{j k}^{1} \frac{\left\langle\operatorname{grad}_{1}^{g} f_{j}, \operatorname{grad}_{1}^{g} f_{k}\right\rangle}{f_{j} f_{k}}\right) f_{1}^{m_{1}-2} f_{2}^{m_{2}} d \mu^{g}}{\operatorname{Vol}\left(M, \tilde{g}_{1}\right)^{\frac{m-2}{m}}} \\
& +\varepsilon^{2 \frac{m_{1}}{m}} \frac{\int_{M}\left(R_{2}^{g}+\sum_{j, k=1}^{2} b_{j k}^{2} \frac{\left\langle\operatorname{grad}_{2}^{g} f_{j}, \operatorname{grad}_{2}^{g} f_{k}\right\rangle}{f_{j} f_{k}}\right) f_{1}^{m_{1}} f_{2}^{m_{2}-2} d \mu^{g}}{\operatorname{Vol}\left(M, \tilde{g}_{1}\right)^{\frac{m-2}{m}}} \\
& +\varepsilon^{2 \frac{m_{1}}{m}} \sum_{i=3}^{l} \frac{\int_{M} R_{i}^{g} f_{1}^{m_{1}} f_{2}^{m_{2}} d \mu^{g}}{\operatorname{Vol}\left(M, \tilde{g}_{1}\right)^{\frac{m-2}{m}}},
\end{aligned}
$$

where $m=\operatorname{dim}(M)$. Since the inequality (5.1) holds by construction, the first term on the right hand side is negative. Moreover, $\lim _{\varepsilon \rightarrow 0} \varepsilon^{2\left(\frac{m_{1}}{m}-1\right)}=\infty$. Thus,

$$
\lim _{\varepsilon \rightarrow 0} \mu\left(M,\left[\tilde{g}_{\varepsilon}\right)\right) \leq \lim _{\varepsilon \rightarrow 0} E\left(\tilde{g}_{\varepsilon}\right)=-\infty .
$$

\section{Multiconformal metric of permutation type}

In Theorem 1.2 we showed that the multiconformal class $\llbracket g \rrbracket$ of a direct product manifold $(M, g)=\left(M_{1}, g_{1}\right) \times \cdots \times\left(M_{l}, g_{l}\right)$ contains metrics $\tilde{g}$ of strictly negative scalar curvature. This is even the case when $\mu\left(M_{i},\left[g_{i}\right]\right) \geq 0$ for each $i \in\{1, \ldots, l\}$. The goal of this section is to show that in this case, such a negative scalar curvature metric cannot be of permutation type in the sense of Definition 6.1. 
Let $(M, g)=\left(M_{1}, g_{1}\right) \times \cdots \times\left(M_{l}, g_{l}\right)$ be a direct product of Riemannian manifolds. For functions $f_{1}, \ldots, f_{l} \in C_{+}^{\infty}(M)$, we may associate the $(l \times l)$-matrix

$$
\left(\begin{array}{ccc}
d_{1} f_{1} & \cdots & d_{1} f_{l} \\
\vdots & & \vdots \\
d_{l} f_{1} & \cdots & d_{l} f_{l}
\end{array}\right)
$$

of 1 -forms on $M$. In view of this matrix, we impose the following conditions on the multiconformal factors.

Definition 6.1 Let $\tilde{g}=f_{1}^{2} g_{1} \oplus \cdots \oplus f_{l}^{2} g_{l}$. We say that $\tilde{g}$ has off-diagonal type if $f_{i}$ is constant along $M_{i}$ for every $i$. For a map $\sigma:\{1, \ldots, l\} \rightarrow\{1, \ldots, l\}$, we say $\tilde{g}$ has type $\sigma$ if $f_{i}$ only depends on $M_{\sigma(i)}$, i.e. is constant along $M_{1} \times \cdots \times M_{\sigma(i)-1} \times M_{\sigma(i)+1} \times \cdots \times M_{l}$ for every $i$. If $\tilde{g}$ has type $\sigma$ for some bijection $\sigma$, then $\tilde{g}$ is said to have permutation type.

Note that a multiconformal metric $\tilde{g}=f_{1}^{2} g_{1} \oplus \cdots \oplus f_{l}^{2} g_{l}$ has off-diagonal if and only if (6.1) has zero diagonal entries. Similarly, $\tilde{g}$ has permutation type if (6.1) is a generalized permutation matrix.

Remark 6.2 Related terminology is the following. The notion of warped products in the sense of Bishop-O'Neill (cf. [6, §7], [31, § 7]) has been generalized to various situations. We remark that doubly warped products can have two different meanings; some authors ${ }^{3}$ deal only with two factors while others ${ }^{4}$ need three factors to define them. The term multiply warped products seems to be unambiguous ${ }^{5}$, but it conflicts with the first meaning of doubly warped products.

Twisted products in the sense of Chen [9, p. 66], also called umbilic products in earlier work of Bishop [5, p. 27], are defined on direct product manifolds, which are topologically not twisted. Note that Bishop-O'Neill [6, p. 29] used the term warped bundles for the generalization of warped products to (possibly topologically twisted) bundles. These notions are generalized, depending on the authors' preferences, to umbilic products, ${ }^{6}$ twisted products ${ }^{7}$ and doubly or multiply twisted products ${ }^{8}$ etc.

Theorem 6.3 Let $(M, g)=\left(M_{1}, g_{1}\right) \times \cdots \times\left(M_{l}, g_{l}\right)$ be a direct product of closed Riemannian manifolds, and assume $R_{i}^{g} \geq 0$ for every $1 \leq i \leq l$. If a multiconformal metric $\tilde{g}=f_{1}^{2} g_{1} \oplus \cdots \oplus f_{l}^{2} g_{l}$ has permutation type and $R^{\tilde{g}} \leq 0$, then $f_{1}, \ldots, f_{l}$ are constant. In particular, $R^{\tilde{g}} \equiv R^{g} \equiv 0$.

\footnotetext{
3 e.g. Allison [1, Definition 2.2], Yang [53, p. 203], Ünal [48, Definition 2.1], Brozos-Vázquez-García-RíoVázquez-Lorenzo [7, Remark 5], Olteanu [30, Definition 1].

4 e.g. Zucker [54, p. 215], Gromov-Lawson [22, p. 188], Ivey [24], Petersen [33, Chapter 1, § 4], Walsh [50, p. 6].

5 cf. Brüning [8, p. 303], Ünal [47, Definition 2.1], Dobarro-Ünal [13, Definition 2.1], Uğuz-Bilge [49, $\S 2.2]$, Chen [10, p. 13].

6 cf. Gauchman [20, Definition 1].

7 cf. Koike [26, p. 3], Meumertzheim-Reckziegel-Schaaf [28, Definition 2].

8 cf. Ponge-Reckziegel [34, p. 15], Rovenskii [37, Definition 2.6], Fernández-López-García-Río-KupeliÜnal [19, p. 214],

Uddin [46, p. 35], Wang [51, p. 1].
} 
Proof Let $\tilde{g}=f_{1}^{2} g_{1} \oplus \cdots \oplus f_{l}^{2} g_{l}$ has type $\sigma$ for some permutation $\sigma$. We may assume $\sigma(i) \neq i$ for every $i$. Indeed, if $\sigma(j)=j$ for some $j$, then $(M, \tilde{g})$ is isomorphic to

$$
\left(M_{j}, f_{j}^{2} g_{j}\right) \times\left(\prod_{i \neq j} M_{i}, \bigoplus_{i \neq j} f_{i}^{2} g_{i}\right)
$$

Set $m_{i}=\operatorname{dim}\left(M_{i}\right)$ and let $q_{1}, \ldots, g_{l}$ be real numbers to be specified later. By assumption, $f_{i}$ only depends on $M_{\sigma(i)}$ for any $1 \leq i \leq l$. In particular, $\operatorname{grad}_{j}^{g} f_{i}=0$ for all $j \neq \sigma(i)$. Since we can additionally assume $\sigma(i) \neq i$ for all $1 \leq i \leq l$, the integral formula in Proposition 3.3 simplifies to

$$
\begin{aligned}
& \int_{M}\left(R^{\tilde{g}}-\sum_{i=1}^{l} \frac{R_{i}^{g}}{f_{i}^{2}}\right) f_{1}^{q_{1}} \cdots f_{l}^{q_{l}} d \mu^{g} \\
& =\sum_{j \neq i} m_{j}\left(2 q_{j}-m_{j}-1\right) \int_{M} \frac{\left|\operatorname{grad}_{i}^{g} f_{j}\right|_{g}^{2}}{f_{i}^{2} f_{j}^{2}} f_{1}^{q_{1}} \cdots f_{l}^{q_{l}} d \mu^{g} \\
& \quad+\sum_{i \neq j, k \neq i, j \neq k}\left(m_{j} q_{k}+q_{j} m_{k}-m_{j} m_{k}\right) \int_{M} \frac{\left\langle\operatorname{grad}_{i}^{g} f_{j}, \operatorname{grad}_{i}^{g} f_{k}\right\rangle}{f_{i}^{2} f_{j} f_{k}} f_{1}^{q_{1}} \cdots f_{l}^{q_{l}} d \mu^{g}(6.2) \\
& =\sum_{i=1}^{l}\left(m_{i}\left(2 q_{i}-m_{i}-1\right)+\left(2 m_{i} q_{i}-m_{i}^{2}\right)\right) \int_{M} \frac{\left|\operatorname{grad}_{\sigma(i)}^{g} f_{i}\right|_{g}^{2}}{f_{\sigma(i)}^{2} f_{i}} f_{1}^{q_{1}} \cdots f_{l}^{q_{l}} d \mu^{g} \\
& =\sum_{i=1}^{l}\left(4 m_{i} q_{i}-2 m_{i}^{2}-m_{i}\right) \int_{M} \frac{\left|\operatorname{grad}_{\sigma(i)}^{g} f_{i}\right|_{g}^{2}}{f_{\sigma(i)}^{2} f_{i}} f_{1}^{q_{1}} \cdots f_{l}^{q_{l}} d \mu^{g} .
\end{aligned}
$$

For $q_{i}=m_{i}$ the right hand side of (6.2) is nonnegative and zero if and only if $f_{1}, \ldots, f_{l}$ are constant, implying that

$$
\int_{M} R^{\tilde{g}} f_{1}^{m_{1}} \cdots f_{l}^{m_{l}} d \mu^{g} \geq \int_{M} \sum_{i=1}^{l} \frac{R_{i}^{g}}{f_{i}^{2}} f_{1}^{m_{1}} \cdots f_{l}^{m_{l}} d \mu_{g},
$$

with equality if and only if $f_{1}, \ldots, f_{l}$ are constant. Since $\sum_{i=1}^{l} \frac{R_{i}^{g}}{f_{i}^{2}}$ is by assumption nonnegative the claim follows.

Yang [53, Theorem 1] observed that a $\left(M_{1} \times M_{2}, \tilde{g}=f_{1}^{2} g_{1} \oplus f_{2}^{2} g_{2}\right)$ is of warped product type with $f_{i}$ nonconstant for $i=1,2$ can only have constant scalar curvature if it is scalar flat. He then asked whether there exist such scalar flat metrics of nontrivial warped product type. Before providing an answer to his question, we generalize Yang's Theorem slightly to our setting as follows.

Theorem 6.4 Let $(M, g)=\left(M_{1}, g_{1}\right) \times \cdots \times\left(M_{l}, g_{l}\right)$ be a direct product of closed connected Riemannian manifolds and let $\tilde{g}=f_{1}^{2} g_{1} \oplus \cdots f_{l}^{2} g_{l}$ be a metric of constant scalar curvature. If $\tilde{g}$ is of permutation type for a bijection $\sigma$ with $\sigma(i) \neq i$ for all $1 \leq i \leq l$ and the functions $f_{1}, \ldots, f_{l}$ are nonconstant then $R^{\tilde{g}}=0$. 
Proof As, by assumption $\operatorname{grad}_{i} f_{i}=0$ for all $1 \leq i \leq l$ the formula for the scalar curvature given in Theorem 3.1 simplifies to

$$
\begin{aligned}
R^{\tilde{g}} & =\sum_{i} \frac{R_{i}^{g}}{f_{i}^{2}}-2 \sum_{i \neq j} m_{j} \frac{\Delta_{i}^{g} f_{j}}{f_{i}^{2} f_{j}}-\sum_{i \neq j} m_{j}\left(m_{j}-1\right) \frac{\left|\operatorname{grad}_{i}^{g} f_{j}\right|^{2}}{f_{i}^{2} f_{j}^{2}} \\
& =\sum_{i=1}^{l} \frac{1}{f_{i}^{2}}\left(R_{i}^{g}-2 m_{\sigma^{-1}(i)} \frac{\Delta_{i}^{g} f_{\sigma^{-1}(i)}}{f_{\sigma^{-1}(i)}}-m_{\sigma^{-1}(i)}\left(m_{\sigma^{-1}(i)}-1\right) \frac{\left|\operatorname{grad}_{i}^{g} f_{\sigma^{-1}(i)}\right|^{2}}{f_{\sigma^{-1}(i)}^{2}}\right) .
\end{aligned}
$$

We fix $i \in\{1, \ldots, l\}$ and take a vector field $X$ tangent to $E_{\sigma(i)}$. Differentiation of (6.3) with respect to $X$ yields

$$
\begin{aligned}
0= & X\left(R^{\tilde{g}}\right) \\
= & X\left(\frac{1}{f_{i}^{2}}\right) \cdot\left(R_{i}^{g}-2 m_{\sigma^{-1}(i)} \frac{\Delta_{i}^{g} f_{\sigma^{-1}(i)}}{f_{\sigma^{-1}(i)}}-m_{\sigma^{-1}(i)}\left(m_{\sigma^{-1}(i)}-1\right) \frac{\left|\operatorname{grad}_{i}^{g} f_{\sigma^{-1}(i)}\right|^{2}}{f_{\sigma^{-1}(i)}^{2}}\right) \\
& +\frac{1}{f_{\sigma(i)}^{2}} X\left(R_{\sigma(i)}^{g}-2 m_{i} \frac{\Delta_{\sigma(i)}^{g} f_{i}}{f_{i}}-m_{i}\left(m_{i}-1\right) \frac{\left|\operatorname{grad}_{\sigma(i)}^{g} f_{i}\right|^{2}}{f_{i}^{2}}\right) .
\end{aligned}
$$

In other words,

$$
\begin{aligned}
& X\left(\frac{1}{f_{i}^{2}}\right) \cdot f_{\sigma(i)}^{2}\left(R_{i}^{g}-2 m_{\sigma^{-1}(i)} \frac{\Delta_{i}^{g} f_{\sigma^{-1}(i)}}{f_{\sigma^{-1}(i)}}-m_{\sigma^{-1}(i)}\left(m_{\sigma^{-1}(i)}-1\right) \frac{\left|\operatorname{grad}_{i}^{g} f_{\sigma^{-1}(i)}\right|^{2}}{f_{\sigma^{-1}(i)}^{2}}\right) \\
& \quad=-X\left(R_{\sigma(i)}^{g}-2 m_{i} \frac{\Delta_{\sigma(i)}^{g} f_{i}}{f_{i}}-m_{i}\left(m_{i}-1\right) \frac{\left|\operatorname{grad}_{\sigma(i)}^{g} f_{i}\right|^{2}}{f_{i}^{2}}\right) .
\end{aligned}
$$

By assumptions, the right hand side of (6.4) only depends on $M_{\sigma(i)}$. Since,

$$
f_{\sigma(i)}^{2}\left(R_{i}^{g}-2 m_{\sigma^{-1}(i)} \frac{\Delta_{i}^{g} f_{\sigma^{-1}(i)}}{f_{\sigma^{-1}(i)}}-m_{\sigma^{-1}(i)}\left(m_{\sigma^{-1}(i)}-1\right) \frac{\left|\operatorname{grad}_{i}^{g} f_{\sigma^{-1}(i)}\right|^{2}}{f_{\sigma^{-1}(i)}^{2}}\right)
$$

is constant along $M_{\sigma(i)}$, there are only two possibilities: Either (6.5) is constant or $X\left(\frac{1}{f_{i}^{2}}\right) \equiv 0$. But, by assumption, $\frac{1}{f_{i}^{2}}$ is nonconstant along $M_{\sigma(i)}$. Therefore, we can choose $X$ such that $X\left(\frac{1}{f_{i}^{2}}\right)$ does not vanish everywhere. Hence, there is a $c_{i} \in \mathbb{R}$ such that

$$
f_{\sigma(i)}^{2}\left(R_{i}^{g}-2 m_{\sigma^{-1}(i)} \frac{\Delta_{i}^{g} f_{\sigma^{-1}(i)}}{f_{\sigma^{-1}(i)}}-m_{\sigma^{-1}(i)}\left(m_{\sigma^{-1}(i)}-1\right) \frac{\left|\operatorname{grad}_{i}^{g} f_{\sigma^{-1}(i)}\right|^{2}}{f_{\sigma^{-1}(i)}^{2}}\right) \equiv c_{i} .
$$

Combining this observation with (6.3) we obtain

$$
R^{\tilde{g}}=\sum_{i=1}^{l} \frac{c_{i}}{f_{i}^{2} f_{\sigma(i)}^{2}}
$$


As above, we choose a vector field $X$ tangent to $M_{\sigma(i)}$ such that $X\left(\frac{1}{f_{i}^{2}}\right)$ does not vanish everywhere. Differentiation with respect to this vector field leads to

$$
0=X\left(R^{\tilde{g}}\right)=X\left(\frac{1}{f_{i}^{2}}\right)\left(\frac{c_{i}}{f_{\sigma(i)}^{2}}+\frac{c_{\sigma^{-1}(i)}}{f_{\sigma^{-1}(i)}^{2}}\right) .
$$

As $\left(\frac{c_{i}}{f_{\sigma(i)}^{2}}+\frac{c_{\sigma^{-1}(i)}}{f_{\sigma^{-1}(i)}^{2}}\right)$ is constant along $M_{\sigma(i)}$, the above equation implies that

$$
\frac{c_{i}}{f_{\sigma(i)}^{2}}+\frac{c_{\sigma^{-1}(i)}}{f_{\sigma^{-1}(i)}^{2}} \equiv 0
$$

for each $i \in\{1, \ldots, l\}$. Since each $f_{i}$ is nonconstant and only depends on $M_{\sigma(i)}$ (6.6) it follows that $c_{i}=0$ for all $i$. Hence, $R^{\tilde{g}}=0$.

In the spirit of Yang, we ask the following question:

Let $(M, g)=\left(M_{1}, g_{1}\right) \times \cdots \times\left(M_{l}, g_{l}\right)$ be a direct product of closed Riemannian manifolds. Does $M$ admit a scalar flat metric of nontrivial warped product type?

Theorems 1.1 and 6.3 provide the following partial answer: If either $\mu\left(M_{i},\left[g_{i}\right]\right) \leq 0$ for all $1 \leq i \leq l$ or $R_{i}^{g}>0$ for all $1 \leq i \leq l$ each scalar flat metric has to be of product type.

Acknowledgements We thank Professors Bernd Ammann and Naoyuki Koike for their comments on the previous version of this article. N. Otoba is supported by the DFG (Deutsche Forschungsgemeinschaft), SFB 1085 Higher Invariants. S. Roos was supported by the Hausdorff Center for Mathematics in Bonn.

Funding Open Access funding enabled and organized by Projekt DEAL.

Open Access This article is licensed under a Creative Commons Attribution 4.0 International License, which permits use, sharing, adaptation, distribution and reproduction in any medium or format, as long as you give appropriate credit to the original author(s) and the source, provide a link to the Creative Commons licence, and indicate if changes were made. The images or other third party material in this article are included in the article's Creative Commons licence, unless indicated otherwise in a credit line to the material. If material is not included in the article's Creative Commons licence and your intended use is not permitted by statutory regulation or exceeds the permitted use, you will need to obtain permission directly from the copyright holder. To view a copy of this licence, visit http://creativecommons.org/licenses/by/4.0/.

\section{Appendix}

\section{A Computation of the scalar curvature}

In this appendix we present a detailed proof of Theorem 3.1. Thus, let $M$ be an almost product manifold with decomposition $T M=\bigoplus_{i=1}^{l} E_{i}$ with $m_{i}:=\operatorname{rank}\left(E_{i}\right)$. Further we fix a compatible metric $g$ and denote by $\mathcal{P}_{i}: T M \rightarrow E_{i}$ the corresponding orthogonal projections. Recall the following definitions from (3.1):

$$
\begin{aligned}
d_{i} f(X) & :=d f\left(\mathcal{P}_{i} X\right) \\
\operatorname{grad}_{i}^{g} f & :=\mathcal{P}_{i} \operatorname{grad}^{g}(f), \\
\operatorname{Hess}_{i}^{g} f(X, Y) & :=\operatorname{Hess}^{g} f\left(\mathcal{P}_{i}(X), \mathcal{P}_{i} Y\right), \\
\Delta_{i}^{g} f & :=\operatorname{tr}^{g} \operatorname{Hess}_{i}^{g} f .
\end{aligned}
$$


for each $f \in C^{\infty}(M)$ and $X, Y \in T M$ and fix a multiconformal change $\tilde{g}=f_{1}^{2} g_{1} \oplus \cdots \oplus$ $f_{l}^{2} g_{l}$. Henceforth, we write $\tilde{g}=\langle\langle\cdot, \cdot\rangle\rangle$ and $g=\langle\cdot, \cdot\rangle$.

Before we start computing the scalar curvature under a multiconformal change, we first relate the Levi-Civita connections $\nabla^{g}, \nabla^{\tilde{g}}$ of $g, \tilde{g}$, respectively. As the derivatives of the multiconformal factors $f_{1}, \ldots, f_{l}$ will be involved it is reasonable to first compare the gradients taken with respect to the metrics $g$ and $\tilde{g}$.

Lemma A.1 For every $\varphi \in C^{\infty}(M)$,

$$
\operatorname{grad}^{\tilde{g}} \varphi=\sum_{a=1}^{l} f_{a}^{-2} \operatorname{grad}_{a}^{g} \varphi .
$$

Proof For every $X \in \Gamma(T M)$, we can express the derivative $X(\varphi)$ either with respect to $g$ or with respect to $\tilde{g}$. This leads to,

$$
\begin{aligned}
& X(\varphi)=\left\langle\left\langle\operatorname{grad}^{\tilde{g}} \varphi, X\right\rangle\right\rangle, \\
& X(\varphi)=\left\langle\operatorname{grad}^{g} \varphi, X\right\rangle=\sum_{i=1}^{l}\left\langle\left\langle f_{i}^{-2} \mathcal{P}_{i} \cdot \operatorname{grad}^{g} \varphi, X\right\rangle\right\rangle .
\end{aligned}
$$

As $X(\varphi)$ is independent of the Riemannian metric, (A.2) holds.

Proposition A.2 Define $T_{X} Y=\nabla_{X}^{\tilde{g}} Y-\nabla_{X}^{g} Y$ for $X, Y \in \Gamma(T M)$. Then

$$
\begin{aligned}
T_{X} Y= & \sum_{a=1}^{l}\left\langle X, \operatorname{grad}^{g} f_{a}\right\rangle \frac{1}{f_{a}} \mathcal{P}_{a} Y+\sum_{a=1}^{l}\left\langle Y, \operatorname{grad}^{g} f_{a}\right\rangle \frac{1}{f_{a}} \mathcal{P}_{i} X \\
& -\sum_{a, b=1}^{l}\left\langle\mathcal{P}_{b} X, \mathcal{P}_{b} Y\right\rangle \frac{f_{b}}{f_{a}^{2}} \operatorname{grad}_{a}^{g} f_{b} .
\end{aligned}
$$

Proof Since $T$ is tensorial, we assume without loss of generality that $X \in \Gamma\left(E_{i}\right), Y \in \Gamma\left(E_{j}\right)$. Comparing the Koszul formulas

$$
\begin{aligned}
2\left\langle\left\langle\nabla_{X}^{\tilde{g}} Y, Z\right\rangle\right\rangle= & X\langle\langle Y, Z\rangle\rangle+Y\langle\langle X, Z\rangle\rangle-Z\langle\langle X, Y\rangle\rangle \\
& -\langle\langle X,[Y, Z]\rangle-\langle\langle Y,[X, Z]\rangle\rangle+\langle\langle Z,[X, Y]\rangle, \\
2\left\langle\nabla_{X}^{g} Y, Z\right\rangle= & X\langle Y, Z\rangle+Y\langle X, Z\rangle-Z\langle X, Y\rangle \\
& -\langle X,[Y, Z]\rangle-\langle Y,[X, Z]\rangle+\langle Z,[X, Y]\rangle
\end{aligned}
$$

for $\tilde{g}$ and $g$, we observe that if $Z \in \Gamma\left(E_{a}\right)$ then

$$
\begin{aligned}
2 f_{a}^{2}\left\langle\nabla_{X}^{\tilde{g}} Y, Z\right\rangle= & 2\left\langle\left\langle\nabla_{X}^{\tilde{g}} Y, Z\right\rangle\right\rangle \\
= & \left.X\left(f_{a}^{2}\langle Y, Z\rangle\right)+Y\left(f_{a}^{2}\langle X, Z\rangle\right)-Z\langle X, Y\rangle\right\rangle \\
& -f_{a}^{2}\langle X,[Y, Z]\rangle-f_{a}^{2}\langle Y,[X, Z]\rangle+f_{a}^{2}\langle Z,[X, Y]\rangle \\
= & 2 f_{a} X\left(f_{a}\right)\langle Y, Z\rangle+2 f_{a} Y\left(f_{a}\right)\langle X, Z\rangle-Z\langle\langle X, Y\rangle\rangle \\
& +f_{a}^{2} X\langle Y, Z\rangle+f_{a}^{2} Y\langle X, Z\rangle \\
& -f_{a}^{2} Z\langle X, Y\rangle+f_{a}^{2} Z\langle X, Y\rangle \\
& -f_{a}^{2}\langle X,[Y, Z]\rangle-f_{a}^{2}\langle Y,[X, Z]\rangle+f_{a}^{2}\langle Z,[X, Y]\rangle \\
= & 2 f_{a}^{2}\left\langle\nabla_{X}^{g} Y, Z\right\rangle
\end{aligned}
$$




$$
\begin{aligned}
& +2 f_{a} X\left(f_{a}\right)\langle Y, Z\rangle+2 f_{a} Y\left(f_{a}\right)\langle X, Z\rangle \\
& -Z\langle\langle X, Y\rangle\rangle+f_{a}^{2} Z\langle X, Y\rangle .
\end{aligned}
$$

Dividing both sides by $2 f_{a}^{2}$, we obtain

$$
\left\langle\nabla_{X}^{\tilde{g}} Y-\nabla_{X}^{g} Y, Z\right\rangle=\frac{X\left(f_{a}\right)}{f_{a}}\langle Y, Z\rangle+\frac{Y\left(f_{a}\right)}{f_{a}}\langle X, Z\rangle-\frac{1}{2} \frac{Z}{f_{a}^{2}}\langle\langle X, Y\rangle\rangle+\frac{1}{2} Z\langle X, Y\rangle .
$$

Hence,

$$
\begin{aligned}
T_{X} Y= & \nabla_{X}^{\tilde{g}} Y-\nabla_{X}^{g} Y \\
= & \sum_{a=1}^{l} \frac{X\left(f_{a}\right)}{f_{a}} \mathcal{P}_{a} Y+\sum_{a=1}^{l} \frac{Y\left(f_{a}\right)}{f_{a}} \mathcal{P}_{a} X-\frac{1}{2} \operatorname{grad}^{\tilde{g}}\langle\langle X, Y\rangle\rangle+\frac{1}{2} \operatorname{grad}^{g}\langle X, Y\rangle \\
= & \sum_{a=1}^{l}\left\langle X, \operatorname{grad}^{g} f_{a}\right\rangle \frac{1}{f_{a}} \mathcal{P}_{a} Y+\sum_{a=1}^{l}\left\langle Y, \operatorname{grad}^{g} f_{a}\right\rangle \frac{1}{f_{a}} \mathcal{P}_{a} X \\
& -\frac{1}{2} \operatorname{grad}^{\tilde{g}}\langle\langle X, Y\rangle\rangle+\frac{1}{2} \operatorname{grad}^{g}\langle X, Y\rangle .
\end{aligned}
$$

To conclude the claimed formula it remains to show that

$$
-\frac{1}{2} \operatorname{grad}^{\tilde{g}}\langle\langle X, Y\rangle\rangle+\frac{1}{2} \operatorname{grad}^{g}\langle X, Y\rangle=-\sum_{a, b=1}^{l}\left\langle\mathcal{P}_{b} X, \mathcal{P}_{b} Y\right\rangle \frac{f_{b}}{f_{a}^{2}} \mathcal{P}_{a} \operatorname{grad}^{g} f_{b}
$$

for $X, Y \in \Gamma(T M)$.

The term $-\frac{1}{2} \operatorname{grad}^{\tilde{g}}\langle\langle X, Y\rangle\rangle+\frac{1}{2} \operatorname{grad}^{g}\langle X, Y\rangle$ is tensorial, since it is the difference of two tensors $T_{X} Y$ and $\sum_{a=1}^{l}\left\langle X, \operatorname{grad}^{g} f_{a}\right\rangle \frac{1}{f_{a}} \mathcal{P}_{a} Y+\sum_{a=1}^{l}\left\langle Y\right.$, $\left.\operatorname{grad}^{g} f_{a}\right\rangle \frac{1}{f_{a}} \mathcal{P}_{a} X$. Thus, it suffices to check (A.4) for an $g$-orthonormal frame $\left\{e_{\alpha}\right\}_{\alpha=1}^{m}$ such that for every $\alpha \in\{1, \ldots, m\}$ there exists some $i \in\{1, \ldots, l\}$ with $e_{\alpha} \in \Gamma\left(E_{i}\right)$. Since $\left\{e_{\alpha}\right\}_{\alpha=1}^{m}$ remains orthogonal with respect to $\tilde{g}$, (A.4) holds for $X=e_{\alpha}, Y=e_{\beta}$ if $\alpha \neq \beta$ as both sides evaluate to 0 . If $\alpha=\beta$, we derive for the left hand side

$$
\begin{aligned}
-\frac{1}{2} \operatorname{grad}^{\tilde{g}}\left\langle\left\langle e_{\alpha}, e_{\alpha}\right\rangle\right\rangle+\frac{1}{2} \operatorname{grad}^{g}\left\langle e_{\alpha}, e_{\alpha}\right\rangle & =-\frac{1}{2} \operatorname{grad}^{\tilde{g}}\left(f_{i}^{2}\right) \\
& =-\frac{1}{2} \sum_{a=1}^{l} f_{a}^{-2} \mathcal{P}_{a} \operatorname{grad}^{g}\left(f_{i}^{2}\right)=-\sum_{a=1}^{l} \frac{f_{i}}{f_{a}^{2}} \mathcal{P}_{a} \operatorname{grad}^{g} f_{i},
\end{aligned}
$$

and for the right hand side

$$
-\sum_{a, b=1}^{l}\left\langle\mathcal{P}_{b} e_{\alpha}, \mathcal{P}_{b} e_{\alpha}\right\rangle \frac{f_{b}}{f_{a}^{2}} \mathcal{P}_{a} \operatorname{grad}^{g} f_{b}=-\sum_{a=1}^{l} \frac{f_{i}}{f_{a}^{2}} \mathcal{P}_{a} \operatorname{grad} f_{i} .
$$

Now (A.4) follows by combining these two identities.

These relations are sufficient to derive a formula for the scalar curvature under a multiconformal change.

Theorem A.3 The scalar curvature of $R^{\tilde{g}}$ satisfies

$$
R^{\tilde{g}}=\sum_{i=1}^{l} \frac{R_{i}^{g}}{f_{i}^{2}}+\sum_{i=1}^{l} \frac{\rho_{i}}{f_{i}^{2}}
$$


where $\rho_{i}$ is defined by

$$
\begin{aligned}
\rho_{i}= & \rho_{i}^{g}\left(f_{1}, \ldots, f_{l}\right) \\
= & -2\left(m_{i}-1\right) \frac{\Delta_{i}^{g} f_{i}}{f_{i}}-2 \sum_{j \neq i} m_{j} \frac{\Delta_{i}^{g} f_{j}}{f_{j}} \\
& -\left(m_{i}-1\right)\left(m_{i}-4\right) \frac{\left|\operatorname{grad}_{i}^{g} f_{i}\right|_{g}^{2}}{f_{i}^{2}}-2\left(m_{i}-2\right) \sum_{j \neq i} m_{j} \frac{g\left(\operatorname{grad}_{i}^{g} f_{i}, \operatorname{grad}_{i}^{g} f_{j}\right)}{f_{i} f_{j}} \\
& -\sum_{j \neq i} m_{j}\left(m_{j}-1\right) \frac{\left|\operatorname{grad}_{i}^{g} f_{j}\right|_{g}^{2}}{f_{j}^{2}}-\sum_{j \neq i, k \neq i, j \neq k} m_{j} m_{k} \frac{g\left(\operatorname{grad}_{i}^{g} f_{j}, \operatorname{grad}_{i}^{g} f_{k}\right)}{f_{j} f_{k}},
\end{aligned}
$$

Proof To begin with, we recall the general formula

$$
R^{\tilde{g}}(X, Y) Z=R(X, Y) Z+\left(\nabla_{X} T\right)_{Y} Z-\left(\nabla_{Y} T\right)_{X} Z+T_{X} T_{Y} Z-T_{Y} T_{X} Z
$$

for $X, Y, Z \in \Gamma(T M)$, which can be shown by summing up the following three identities

$$
\begin{aligned}
-\nabla_{[X, Y]}^{\tilde{g}} Z & =-\nabla_{[X, Y]} Z-T_{[X, Y]} Z, \\
-\nabla_{Y}^{\tilde{g}} \nabla_{X}^{\tilde{g}} Z & =-\nabla_{Y}^{\tilde{g}}\left(\nabla_{X} Z+T_{X} Z\right) \\
& =-\nabla_{Y} \nabla_{X} Z-\left(\nabla_{Y} T\right)_{X} Z-T_{\nabla_{Y} X} Z-T_{X}\left(\nabla_{Y} Z\right)-T_{Y}\left(\nabla_{X} Z\right)-T_{Y} T_{X} Z, \\
\nabla_{X}^{\tilde{g}} \nabla_{Y}^{\tilde{g}} Z & =\nabla_{X} \nabla_{Y} Z+\left(\nabla_{X} T\right)_{Y} Z+T_{\nabla_{X} Y} Z+T_{Y}\left(\nabla_{X} Z\right)+T_{X}\left(\nabla_{Y} Z\right)+T_{X} T_{Y} Z .
\end{aligned}
$$

Here and henceforth in the proof, quantities without any superscript such as $R$ and $\nabla$ are understood to be the ones with respect to $g$.

We want to express all these identities in terms of the functions $f_{1}, \ldots, f_{l}$ and their derivatives. Let $X \in \Gamma\left(E_{i}\right), Y \in \Gamma\left(E_{j}\right), Z \in \Gamma\left(E_{k}\right)$. Then (A.3) yields

$$
\begin{aligned}
& T_{X} Y=\left\langle X, \operatorname{grad} f_{j}\right\rangle \frac{1}{f_{j}} Y+\left\langle Y, \operatorname{grad} f_{i}\right\rangle \frac{1}{f_{i}} X-\langle X, Y\rangle \sum_{a=1}^{l} \frac{f_{i}}{f_{a}^{2}} \mathcal{P}_{a} \operatorname{grad} f_{i}, \\
& T_{Y} Z=\left\langle Y, \operatorname{grad} f_{k}\right\rangle \frac{1}{f_{k}} Z+\left\langle Z, \operatorname{grad} f_{j}\right\rangle \frac{1}{f_{j}} Y-\langle Y, Z\rangle \sum_{a=1}^{l} \frac{f_{j}}{f_{a}^{2}} \mathcal{P}_{a} \operatorname{grad} f_{j} .
\end{aligned}
$$

Up to interchanging the roles of $X$ and $Y$ there are two terms that we need to take care of. Namely, $\left(\nabla_{X} T\right)_{Y} Z$ and $T_{X} T_{Y} Z$

On the one hand, (A.8) and (A.3) yields

$$
\begin{aligned}
\left(\nabla_{X} T\right)_{Y} Z= & \nabla_{X}\left(T_{Y} Z\right)-T_{\nabla_{X} Y} Z-T_{Y}\left(\nabla_{X} Z\right) \\
= & \left\langle Y, \nabla_{X} \operatorname{grad} f_{k}\right\rangle \frac{1}{f_{k}} Z-\left\langle Y, \operatorname{grad} f_{k}\right\rangle \frac{X\left(f_{k}\right)}{f_{k}^{2}} Z \\
& +\left\langle Z, \nabla_{X} \operatorname{grad} f_{j}\right\rangle \frac{1}{f_{j}} Y-\left\langle Z, \operatorname{grad} f_{j}\right\rangle \frac{X\left(f_{j}\right)}{f_{j}} Y \\
& -\langle Y, Z\rangle \sum_{a=1}^{l}\left(\frac{X\left(f_{j}\right)}{f_{a}^{2}}-\frac{2 f_{j} X\left(f_{a}\right)}{f_{a}^{3}}\right) \mathcal{P}_{a} \operatorname{grad} f_{j} \\
& -\langle Y, Z\rangle \sum_{a=1}^{l} \frac{f_{j}}{f_{a}^{2}} \mathcal{P}_{a} \operatorname{grad} f_{j}
\end{aligned}
$$




$$
\begin{aligned}
& =\text { Hess } f_{k}(X, Y) \frac{1}{f_{k}} Z-\left\langle X, \operatorname{grad} f_{k}\right\rangle\left\langle Y, \operatorname{grad} f_{k}\right\rangle \frac{1}{f_{k}^{2}} Z \\
& +\operatorname{Hess} f_{j}(X, Z) \frac{1}{f_{j}} Y-\left\langle X, \operatorname{grad} f_{j}\right\rangle\left\langle Z, \operatorname{grad} f_{j}\right\rangle \frac{1}{f_{j}^{2}} Y \\
& \quad-\langle Y, Z\rangle\left\langle X, \operatorname{grad} f_{j}\right\rangle \sum_{a=1}^{l} \frac{1}{f_{a}^{2}} \mathcal{P}_{a} \operatorname{grad} f_{j} \\
& +2\langle Y, Z\rangle \sum_{a=1}^{l}\left\langle X, \operatorname{grad} f_{a}\right\rangle \frac{f_{j}}{f_{a}^{3}} \mathcal{P}_{a} \operatorname{grad} f_{j} \\
& \\
& -\langle Y, Z\rangle \sum_{a=1}^{l} \frac{f_{j}}{f_{a}^{2}} \mathcal{P}_{a} \nabla_{X} \operatorname{grad} f_{j} .
\end{aligned}
$$

Taking the inner product with $W \in \Gamma\left(E_{h}\right)$ leads to,

$$
\begin{aligned}
\left\langle\nabla_{X} T_{Y} Z, W\right\rangle \\
=\langle Z, W\rangle \text { Hess } f_{k}(X, Y) \frac{1}{f_{k}}-\langle Z, W\rangle\left\langle X, \operatorname{grad} f_{k}\right\rangle\left\langle Y, \operatorname{grad} f_{k}\right\rangle \frac{1}{f_{k}^{2}} \\
+\langle Y, W\rangle \text { Hess } f_{j}(X, Z) \frac{1}{f_{j}}-\langle Y, W\rangle\left\langle X, \operatorname{grad} f_{j}\right\rangle\left\langle Z, \operatorname{grad} f_{j}\right\rangle \frac{1}{f_{j}^{2}} \\
\quad-\langle Y, Z\rangle\left\langle X, \operatorname{grad} f_{j}\right\rangle\left\langle W, \operatorname{grad} f_{j}\right\rangle \frac{1}{f_{h}^{2}} \\
+2\langle Y, Z\rangle\left\langle X, \operatorname{grad} f_{h}\right\rangle\left\langle W, \operatorname{grad} f_{j}\right\rangle \frac{f_{j}}{f_{h}^{3}}-\langle Y, Z\rangle \operatorname{Hess} f_{j}(X, W) \frac{f_{j}}{f_{h}^{2}} .
\end{aligned}
$$

On the other hand, plug (A.8) into (A.7) to get

$$
\begin{aligned}
T_{X} T_{Y} Z= & \sum_{a=1}^{l}\left\langle X, \operatorname{grad} f_{a}\right\rangle \frac{1}{f_{a}} \mathcal{P}_{a} T_{Y} Z+\left\langle T_{Y} Z, \operatorname{grad} f_{i}\right\rangle \frac{1}{f_{i}} X \\
& -\sum_{a=1}^{l}\left\langle X, T_{Y} Z\right\rangle \frac{f_{i}}{f_{a}^{2}} \mathcal{P}_{a} \operatorname{grad} f_{i} \\
= & \left\langle X, \operatorname{grad} f_{k}\right\rangle\left\langle Y, \operatorname{grad} f_{k}\right\rangle \frac{1}{f_{k}^{2}} Z+\left\langle X, \operatorname{grad} f_{j}\right\rangle\left\langle Z, \operatorname{grad} f_{j}\right\rangle \frac{1}{f_{j}^{2}} Y \\
& -\langle Y, Z\rangle \sum_{a=1}^{l}\left\langle X, \operatorname{grad} f_{a}\right\rangle \frac{f_{j}}{f_{a}^{3}} \mathcal{P}_{a} \operatorname{grad} f_{j} \\
& +\left\langle Y, \operatorname{grad} f_{k}\right\rangle\left\langle Z, \operatorname{grad} f_{i}\right\rangle \frac{1}{f_{i} f_{k}} X+\left\langle Y, \operatorname{grad} f_{i}\right\rangle\left\langle Z, \operatorname{grad} f_{j}\right\rangle \frac{1}{f_{i} f_{j}} X \\
& -\langle Y, Z\rangle \sum_{c=1}^{l}\left\langle\mathcal{P}_{c} \operatorname{grad} f_{j}, \operatorname{grad} f_{i}\right\rangle \frac{f_{j}}{f_{c}^{2} f_{i}} X \\
& -\langle X, Z\rangle\left\langle Y, \operatorname{grad} f_{k}\right\rangle \sum_{a=1}^{l} \frac{f_{i}}{f_{a}^{2} f_{k}} \mathcal{P}_{a} \operatorname{grad} f_{i}
\end{aligned}
$$




$$
\begin{aligned}
& -\langle X, Y\rangle\left\langle Z, \operatorname{grad} f_{j}\right\rangle \sum_{a=1}^{l} \frac{f_{i}}{f_{a}^{2} f_{j}} \mathcal{P}_{a} \operatorname{grad} f_{i} \\
& +\langle Y, Z\rangle\left\langle X, \operatorname{grad} f_{j}\right\rangle \sum_{a=1}^{l} \frac{f_{j}}{f_{a}^{2} f_{i}} \mathcal{P}_{a} \operatorname{grad} f_{i} .
\end{aligned}
$$

Taking the inner product with $W$,

$$
\begin{aligned}
& \left\langle T_{X} T_{Y} Z, W\right\rangle \\
& =\langle Z, W\rangle\left\langle X, \operatorname{grad} f_{k}\right\rangle\left\langle Y, \operatorname{grad} f_{k}\right\rangle \frac{1}{f_{k}^{2}}+\langle Y, W\rangle\left\langle X, \operatorname{grad} f_{j}\right\rangle\left\langle Z, \operatorname{grad} f_{j}\right\rangle \frac{1}{f_{j}^{2}} \\
& \quad-\langle Y, Z\rangle\left\langle X, \operatorname{grad} f_{h}\right\rangle\left\langle W, \operatorname{grad} f_{j}\right\rangle \frac{f_{j}}{f_{h}^{3}} \\
& \quad+\langle X, W\rangle\left\langle Y, \operatorname{grad} f_{k}\right\rangle\left\langle Z, \operatorname{grad} f_{i}\right\rangle \frac{1}{f_{i} f_{k}}+\langle X, W\rangle\left\langle Y, \operatorname{grad} f_{i}\right\rangle\left\langle Z, \operatorname{grad} f_{j}\right\rangle \frac{1}{f_{i} f_{j}} \\
& \quad-\langle X, W\rangle\langle Y, Z\rangle \sum_{c=1}^{l}\left\langle\operatorname{grad}_{c} f_{j}, \operatorname{grad}_{c} f_{i}\right\rangle \frac{f_{j}}{f_{c}^{2} f_{i}} \\
& \quad-\langle X, Z\rangle\left\langle Y, \operatorname{grad} f_{k}\right\rangle\left\langle W, \operatorname{grad} f_{i}\right\rangle \frac{f_{i}}{f_{h}^{2} f_{k}}-\langle X, Y\rangle\left\langle Z, \operatorname{grad} f_{j}\right\rangle\left\langle W, \operatorname{grad} f_{i}\right\rangle \frac{f_{i}}{f_{h}^{2} f_{j}} \\
& \quad+\langle Y, Z\rangle\left\langle X, \operatorname{grad} f_{j}\right\rangle\left\langle W, \operatorname{grad} f_{i}\right\rangle \frac{f_{j}}{f_{h}^{2} f_{i}} .
\end{aligned}
$$

Therefore, (A.6), (A.9), and (A.10) yields a formula for the difference

$$
\left\langle R^{\tilde{g}}(X, Y) Z-R(X, Y) Z, W\right\rangle
$$

for all $X \in \Gamma\left(E_{i}\right), Y \in \Gamma\left(E_{j}\right), Z \in \Gamma\left(E_{k}\right), W \in \Gamma\left(E_{h}\right)$ and by linearity, it extends to an identity for all vector fields on $M$. However, the resulting formula is a very long expression. As we are interested in a formula for the scalar curvature for a multiconformal change we only consider the difference $\left\langle R^{\tilde{g}}(X, Y) Y-R(X, Y) Y, X\right\rangle$ for $X \in \Gamma\left(E_{i}\right)$ and $Y \in \Gamma\left(E_{j}\right)$. In that case we obtain

$$
\begin{aligned}
& \left\langle R^{\tilde{g}}(X, Y) Y-R(X, Y) Y, X\right\rangle \\
& =\langle X, Y\rangle \operatorname{Hess} f_{j}(X, Y) \frac{1}{f_{j}}+\langle X, Y\rangle \operatorname{Hess} f_{i}(Y, X) \frac{1}{f_{i}} \\
& \quad-\langle X, X\rangle \operatorname{Hess} f_{i}(Y, Y) \frac{1}{f_{i}}-\langle Y, Y\rangle \operatorname{Hess} f_{j}(X, X) \frac{f_{j}}{f_{i}^{2}} \\
& \quad-4\langle X, Y\rangle\left\langle X, \operatorname{grad} f_{i}\right\rangle\left\langle Y, \operatorname{grad} f_{j}\right\rangle \frac{1}{f_{i} f_{j}} \\
& \quad+2\langle Y, Y\rangle\left\langle X, \operatorname{grad} f_{i}\right\rangle\left\langle X, \operatorname{grad} f_{j}\right\rangle \frac{f_{j}}{f_{i}^{3}} \\
& \quad+2\langle X, X\rangle\left\langle Y, \operatorname{grad} f_{i}\right\rangle\left\langle Y, \operatorname{grad} f_{j}\right\rangle \frac{1}{f_{i} f_{j}}
\end{aligned}
$$




$$
\begin{aligned}
& -\langle X, X\rangle\langle Y, Y\rangle \sum_{c=1}^{l}\left\langle\operatorname{grad}_{c} f_{i}, \operatorname{grad}_{c} f_{j}\right\rangle \frac{f_{j}}{f_{c}^{2} f_{i}} \\
& +\langle X, Y\rangle^{2} \sum_{c=1}^{l}\left\langle\operatorname{grad}_{c} f_{i}, \operatorname{grad}_{c} f_{j}\right\rangle \frac{f_{i}}{f_{c}^{2} f_{j}} .
\end{aligned}
$$

Taking an $g$-orthonormal frame $\left\{e_{\alpha}\right\}_{\alpha=1}^{m}$ so that for each $\alpha \in\{1, \ldots, m\}$ there exists some $i=i(\alpha) \in\{1, \ldots, l\}$ with $e_{\alpha} \in \Gamma\left(E_{i}\right)$, we define the associated $\tilde{g}$-orthonormal frame via $\left\{\tilde{e}_{\alpha}=f_{i(\alpha)}^{-1} e_{\alpha}\right\}_{\alpha=1}^{m}$. With respect to these orthonormal frames we conclude

$$
\begin{aligned}
\operatorname{Ric}^{\tilde{g}}(Y, Y)-\operatorname{Ric}(Y, Y) & =\sum_{\alpha=1}^{m}\left\langle\left\langle R^{\tilde{g}}\left(\tilde{e}_{\alpha}, Y\right) Y, \tilde{e}_{\alpha}\right\rangle-\left\langle R\left(e_{\alpha}, Y\right) Y, e_{\alpha}\right\rangle\right. \\
& =\sum_{\alpha=1}^{m}\left\langle R^{\tilde{g}}\left(e_{\alpha}, Y\right) Y-R\left(e_{\alpha}, Y\right) Y, e_{\alpha}\right\rangle \\
& =\sum_{i=1}^{l} \sum_{\alpha}\left\langle R^{\tilde{g}}\left(e_{\alpha}, Y\right) Y-R\left(e_{\alpha}, Y\right) Y, e_{\alpha}\right\rangle
\end{aligned}
$$

Inserting (A.11) leads to

$$
\begin{aligned}
\sum_{i=1}^{l} & \sum_{\alpha}\left\langle R^{\tilde{g}}\left(e_{\alpha}, Y\right) Y-R\left(e_{\alpha}, Y\right) Y, e_{\alpha}\right\rangle \\
& =\sum_{i=1}^{l} \sum_{\alpha}\left\langle e_{\alpha}, Y\right\rangle \operatorname{Hess} f_{j}\left(e_{\alpha}, Y\right) \frac{1}{f_{j}}+\sum_{i=1}^{l} \sum_{\alpha}\left\langle e_{\alpha}, Y\right\rangle \operatorname{Hess} f_{i}\left(Y, e_{\alpha}\right) \frac{1}{f_{i}} \\
& -\sum_{i=1}^{l} \sum_{\alpha} \operatorname{Hess} f_{i}(Y, Y) \frac{1}{f_{i}}-\sum_{i=1}^{l} \sum_{\alpha}|Y|^{2} \operatorname{Hess} f_{j}\left(e_{\alpha}, e_{\alpha}\right) \frac{f_{j}}{f_{i}^{2}} \\
& -4 \sum_{i=1}^{l} \sum_{\alpha}\left\langle e_{\alpha}, Y\right\rangle\left\langle e_{\alpha}, \operatorname{grad}_{i}\right\rangle\left\langle Y, \operatorname{grad} f_{j}\right\rangle \frac{1}{f_{i} f_{j}} \\
& +2 \sum_{i=1}^{l} \sum_{\alpha}|Y|^{2}\left\langle e_{\alpha}, \operatorname{grad}_{i}\right\rangle\left\langle e_{\alpha}, \operatorname{grad}_{j}\right\rangle \frac{f_{j}}{f_{i}^{3}} \\
& +2 \sum_{i=1}^{l} \sum_{\alpha}\left\langle Y, \operatorname{grad}_{i}\right\rangle\left\langle Y, \operatorname{grad}_{j}\right\rangle \frac{1}{f_{i} f_{j}} \\
& -\sum_{i=1}^{l} \sum_{\alpha}|Y|^{2} \sum_{c=1}^{l}\left\langle\operatorname{grad}_{c} f_{i}, \operatorname{grad}_{c} f_{j}\right\rangle \frac{f_{j}}{f_{c}^{2} f_{i}} \\
& +\sum_{i=1}^{l} \sum_{\alpha}\left\langle e_{\alpha}, Y\right\rangle^{2} \sum_{c=1}^{l}\left\langle\operatorname{grad}_{c} f_{i}, \operatorname{grad}_{c} f_{j}\right\rangle \frac{f_{i}}{f_{c}^{2} f_{j}} \\
= & 2 \operatorname{Hess}_{j}(Y, Y) \frac{1}{f_{j}}-\sum_{i=1}^{l} m_{i} \operatorname{Hess}_{i}(Y, Y) \frac{1}{f_{i}}
\end{aligned}
$$




$$
\begin{aligned}
& -\sum_{i=1}^{l}|Y|^{2} \Delta_{i} f_{j} \frac{f_{j}}{f_{i}^{2}}-4 \frac{\left\langle Y, \operatorname{grad} f_{j}\right\rangle^{2}}{f_{j}^{2}} \\
& +2 \sum_{i=1}^{l}|Y|^{2}\left\langle\operatorname{grad}_{i} f_{i}, \operatorname{grad}_{i} f_{j}\right\rangle \frac{f_{j}}{f_{i}^{3}}+2 \sum_{i=1}^{l} m_{i}\left\langle Y, \operatorname{grad} f_{i}\right\rangle\left\langle Y, \operatorname{grad} f_{j}\right\rangle \frac{1}{f_{i} f_{j}} \\
& -\sum_{i=1}^{l} m_{i}|Y|^{2} \sum_{c=1}^{l}\left\langle\operatorname{grad}_{c} f_{i}, \operatorname{grad}_{c} f_{j}\right\rangle \frac{f_{j}}{f_{c}^{2} f_{i}}+|Y|^{2} \sum_{c=1}^{l} \frac{\left|\operatorname{grad}_{c} f_{j}\right|^{2}}{f_{c}^{2}} .
\end{aligned}
$$

We thus obtain

$$
\begin{aligned}
\operatorname{Ric}_{j}^{\tilde{g}}-\operatorname{Ric}_{j}= & 2 \frac{\operatorname{Hess}_{j} f_{j}}{f_{j}}-\sum_{i=1}^{l} m_{i} \frac{\operatorname{Hess}_{j} f_{i}}{f_{i}}-\sum_{i=1}^{l} \Delta_{i} f_{j} \frac{f_{j}}{f_{i}^{2}} g_{j} \\
& -4 \frac{d_{j} f_{j} \otimes d_{j} f_{j}}{f_{j}^{2}}+2 \sum_{i=1}^{l} m_{i} \frac{d_{j} f_{i} \otimes d_{j} f_{j}}{f_{i} f_{j}} \\
& +2 \sum_{i=1}^{l}\left\langle\operatorname{grad}_{i} f_{i}, \operatorname{grad}_{i} f_{j}\right\rangle \frac{f_{j}}{f_{i}^{3}} g_{j} \\
& -\sum_{i=1}^{l} m_{i} \sum_{c=1}^{l}\left\langle\operatorname{grad}_{c} f_{i}, \operatorname{grad}_{c} f_{j}\right\rangle \frac{f_{j}}{f_{c}^{2} f_{i}} g_{j}+\sum_{c=1}^{l} \frac{\left|\operatorname{grad}_{c} f_{j}\right|^{2}}{f_{c}^{2}} g_{j} .
\end{aligned}
$$

Taking the trace with respect to $g$ in (A.12) yields

$$
\begin{aligned}
R_{j}^{\tilde{g}}-\frac{R_{j}}{f_{j}^{2}}= & 2 \frac{\Delta_{j} f_{j}}{f_{j}^{3}}-\sum_{i=1}^{l} m_{i} \frac{\Delta_{j} f_{i}}{f_{j}^{2} f_{i}}-\sum_{i=1}^{l} m_{j} \frac{\Delta_{i} f_{j}}{f_{i}^{2} f_{j}} \\
& -4 \frac{\left|\operatorname{grad}_{j} f_{j}\right|^{2}}{f_{j}^{4}}+2 \sum_{i=1}^{l} m_{i} \frac{\left\langle\operatorname{grad}_{j} f_{i}, \operatorname{grad}_{j} f_{j}\right\rangle}{f_{j}^{3} f_{i}} \\
& +2 \sum_{i=1}^{l} m_{j} \frac{\left\langle\operatorname{grad}_{i} f_{i}, \operatorname{grad}_{i} f_{j}\right\rangle}{f_{i}^{3} f_{j}} \\
& -\sum_{i=1}^{l} m_{i} m_{j} \sum_{c=1}^{l} \frac{\left\langle\operatorname{grad}_{c} f_{i}, \operatorname{grad}_{c} f_{j}\right\rangle}{f_{c}^{2} f_{i} f_{j}}+m_{j} \sum_{c=1}^{l} \frac{\left|\operatorname{grad}_{c} f_{j}\right|^{2}}{f_{c}^{2} f_{j}^{2}}
\end{aligned}
$$

Since $R^{\tilde{g}}=\sum_{j=1}^{l} R_{j}^{\tilde{g}}$ we sum (A.13) over $j \in\{1, \ldots, l\}$ and derive

$$
\begin{aligned}
R^{\tilde{g}} & -\sum_{j=1}^{l} \frac{R_{j}}{f_{j}^{2}} \\
= & 2 \sum_{j=1}^{l} \frac{\Delta_{j} f_{j}}{f_{j}^{3}}-2 \sum_{i, j=1}^{l} m_{i} \frac{\Delta_{j} f_{i}}{f_{j}^{2} f_{i}} \\
& -4 \sum_{j=1}^{l} \frac{\left|\operatorname{grad}_{j} f_{j}\right|^{2}}{f_{j}^{4}}+4 \sum_{i, j=1}^{l} m_{i} \frac{\left\langle\operatorname{grad}_{j} f_{i}, \operatorname{grad}_{j} f_{j}\right\rangle}{f_{j}^{3} f_{i}}
\end{aligned}
$$




$$
\begin{aligned}
& -\sum_{i, j=1}^{l} m_{i} m_{j} \sum_{c=1}^{l} \frac{\left\langle\operatorname{grad}_{c} f_{i}, \operatorname{grad}_{c} f_{j}\right\rangle}{f_{c}^{2} f_{i} f_{j}}+\sum_{j=1}^{l} m_{j} \sum_{c=1}^{l} \frac{\left|\operatorname{grad}_{c} f_{j}\right|^{2}}{f_{c}^{2} f_{j}^{2}} \\
= & -2 \sum_{i=1}^{l}\left(m_{i}-1\right) \frac{\Delta_{i} f_{i}}{f_{i}^{3}}-2 \sum_{i=1}^{l} \sum_{j \neq i} m_{j} \frac{\Delta_{i} f_{j}}{f_{i}^{2} f_{j}} \\
& -\sum_{i=1}^{l}\left(m_{i}-1\right)\left(m_{i}-4\right) \frac{\left|\operatorname{grad}_{i} f_{i}\right|^{2}}{f_{i}^{4}}-2 \sum_{i=1}^{l} \sum_{j \neq i} m_{j}\left(m_{i}-2\right) \frac{\left\langle\operatorname{grad}_{i} f_{i}, \operatorname{grad}_{i} f_{j}\right\rangle}{f_{j}^{3} f_{i}} \\
& -\sum_{i=1}^{l} \sum_{j \neq i} m_{j}\left(m_{j}-1\right) \frac{\left|\operatorname{grad}_{i} f_{j}\right|^{2}}{f_{i}^{2} f_{j}^{2}}-\sum_{i=1}^{l} \sum_{j \neq i, k \neq i, j \neq k} m_{j} m_{k} \frac{\left\langle\operatorname{grad}_{i} f_{j}, \operatorname{grad}_{i} f_{k}\right\rangle}{f_{i}^{2} f_{j} f_{k}} .
\end{aligned}
$$

This is equivalent to the claimed formula for the scalar curvature under a multiconformal change.

\section{References}

1. Allison, D.: Pseudoconvexity in Lorentzian doubly warped products. Geom. Dedicata 39(2), 223-227 (1991)

2. Ammann, B., Madani, F., Pilca, M.: The $S^{1}$-equivariant Yamabe invariant of 3-manifolds. Int. Math. Res. Not. IMRN 20, 6310-6328 (2017)

3. Aubin, T.: Équations différentielles non linéaires et problème de Yamabe concernant la courbure scalaire. J. Math. Pures Appl. (9) 55(3), 269-296 (1976)

4. Bérard-Bergery, L.: Scalar Curvature and Isometry Group, pp. 9-28. Kaigai Publications, Tokyo (1983)

5. Bishop, R.L.: Clairaut submersions. In: Kobayashi, S., Obata, M., Takahashi, T. (eds.) Differential Geometry (in Honor of Kentaro Yano), pp 21-31. Kinokuniya, Tokyo (1972)

6. Bishop, R.L., O’Neill, B.: Manifolds of negative curvature. Trans. Am. Math. Soc. 145, 1-49 (1969)

7. Brozos-Vázquez, M., García-Río, E., Vázquez-Lorenzo, R.: Some remarks on locally conformally flat static space-times. J. Math. Phys. 46(2), 022501, 11 (2005)

8. Brüning, J.: On $L^{2}$-index theorems for complete manifolds of rank-one-type. Duke Math. J. 66(2), 257309 (1992)

9. Chen, B.-Y.: Geometry of Submanifolds and Its Applications. Science University of Tokyo, Tokyo (1981)

10. Chen, B.-Y.: CR-warped submanifolds in Kaehler manifolds. In: Geometry of Cauchy-Riemann submanifolds, pp. 1-25. Springer, Singapore (2016)

11. Danielo, L.: Construction de métriques d'Einstein à partir de transformations biconformes. Ann. Fac. Sci. Toulouse Math. (6) 15(3), 553-588 (2006)

12. Dobarro, F., Lami Dozo, E.: Scalar curvature and warped products of Riemann manifolds. Trans. Am. Math. Soc. 303(1), 161-168 (1987)

13. Dobarro, F., Ünal, B.: Curvature of multiply warped products. J. Geom. Phys. 55(1), 75-106 (2005)

14. Dobarro, F., Ünal, B.: About curvature, conformal metrics and warped products. J. Phys. A 40(46), 13907-13930 (2007)

15. Dobarro, F., Ünal, B.: Curvature in special base conformal warped products. Acta Appl. Math. 104(1), $1-46$ (2008)

16. Ehrlich, P.E., Jung, Y.-T., Kim, S.-B.: Constant scalar curvatures on warped product manifolds. Tsukuba J. Math. 20(1), 239-256 (1996)

17. Ehrlich, P.E., Jung, Y.-T., Kim, S.-B., Shin, C.-G.: Partial differential equations and scalar curvature of warped product manifolds. Nonlinear Anal. 44(4, Ser. A: Theory Methods), 545-553 (2001)

18. Ejiri, N.: Some compact hypersurfaces of constant scalar curvature in a sphere. J. Geom. 19(2), 197-199 (1982)

19. Fernández-López, M., García-Río, E., Kupeli, D.N., Ünal, B.: A curvature condition for a twisted product to be a warped product. Manuscripta Math. 106(2), 213-217 (2001)

20. Gauchman, H.: On a decomposition of Riemannian manifolds. Houston J. Math. 7(3), 365-372 (1981)

21. Gromov, M., Lawson, H.B., Jr.: Spin and scalar curvature in the presence of a fundamental group. I. Ann. Math. (2) 111(2), 209-230 (1980) 
22. Gromov, M., Lawson, H.B., Jr.: Positive scalar curvature and the Dirac operator on complete Riemannian manifolds. Inst. Hautes Études Sci. Publ. Math. 58(83-196), 1983 (1984)

23. Hebey, E., Vaugon, M.: Le problème de Yamabe équivariant. Bull. Sci. Math. 117(2), 241-286 (1993)

24. Ivey, T.: New examples of complete Ricci solitons. Proc. Am. Math. Soc. 122(1), 241-245 (1994)

25. Kazdan, J.L., Warner, F.W.: Existence and conformal deformation of metrics with prescribed Gaussian and scalar curvatures. Ann. Math. 2(101), 317-331 (1975)

26. Koike, N.: Totally umbilic orthogonal nets and decomposition theorems. Saitama Math. J. 10, 1-19 (1992)

27. Leung, M.C.: Conformal deformation of warped products and scalar curvature functions on open manifolds. Bull. Sci. Math. 122(5), 369-398 (1998)

28. Meumertzheim, M., Reckziegel, H., Schaaf, M.: Decomposition of twisted and warped product nets. Results Math. 36(3-4), 297-312 (1999)

29. Mo, X.: Harmonic morphisms via deformation of metrics for horizontally conformal maps. In: Harmonic Morphisms, Harmonic Maps, and Related Topics (Brest, 1997), volume 413 of Chapman \& Hall/CRC Research Notes in Mathematics, pp. 13-21. Chapman \& Hall/CRC, Boca Raton, FL (2000)

30. Olteanu, A.: CR-doubly warped product submanifolds. In: Dragomir, S., Shahid, M., Al-Solamy, Falleh R. (eds.) Geometry of Cauchy-Riemann Submanifolds, pp. 267-288. Springer, Singapore (2016)

31. O’Neill, B.: Semi-Riemannian Geometry, volume 103 of Pure and Applied Mathematics. Academic Press, Inc. [Harcourt Brace Jovanovich], New York (1983). (With applications to relativity)

32. Ou, Y.-L.: $p$-harmonic morphisms, biharmonic morphisms, and nonharmonic biharmonic maps. J. Geom. Phys. 56(3), 358-374 (2006)

33. Petersen, P.: Riemannian Geometry, volume 171 of Graduate Texts in Mathematics, 2nd edn. Springer, New York (2006)

34. Ponge, R., Reckziegel, H.: Twisted products in pseudo-Riemannian geometry. Geom. Dedicata 48(1), 15-25 (1993)

35. Rosenberg, J.: Manifolds of positive scalar curvature: a progress report. In: Surveys in Differential Geometry. Vol. XI, volume 11 of Surveys in Differential Geometry, pp. 259-294. Int. Press, Somerville, MA (2007)

36. Rovenski, V., Zelenko, L.: The mixed Yamabe problem for foliations. Eur. J. Math. 1(3), 503-533 (2015)

37. Rovenskii, V.: Foliations, submanifolds, and mixed curvature. J. Math. Sci. (New York) 99(6), 1699-1787 (2000)

38. Schoen, R.: Conformal deformation of a Riemannian metric to constant scalar curvature. J. Differ. Geom. 20(2), 479-495 (1984)

39. Schoen, R., Yau, S.T.: Existence of incompressible minimal surfaces and the topology of three-dimensional manifolds with nonnegative scalar curvature. Ann. Math. (2) 110(1), 127-142 (1979)

40. Schoen, R., Yau, S.T.: On the structure of manifolds with positive scalar curvature. Manuscripta Math. 28(1-3), 159-183 (1979)

41. Schoen, R., Yau, S.T.: Positive scalar curvature and minimal hypersurface singularities (2017). https:// arxiv.org/abs/1704.05490

42. Slobodeanu, R.: Biconformal changes of metric and pseudo-harmonic morphisms. Rev. Roum. Math. Pures Appl. 50(3), 315-319 (2005)

43. Slobodeanu, R.: A note on higher-charge configurations for the Faddeev-Hopf model. In: Harmonic Maps and Differential Geometry, volume 542 of Contemporary Mathematics, pp. 239-246. Amer. Math. Soc., Providence, RI (2011)

44. Tanno, S.: Conformally flat Riemannian manifolds of constant scalar curvature. Hokkaido Math. J. 9(1), 83-90 (1980)

45. Trudinger, N.S.: Remarks concerning the conformal deformation of Riemannian structures on compact manifolds. Ann. Scuola Norm. Sup. Pisa Cl. Sci. (3) 22, 265-274 (1968)

46. Uddin, S.: On doubly warped and doubly twisted product submanifolds. Int. Electron. J. Geom. 3(1), 35-39 (2010)

47. Ünal, B.: Multiply warped products. J. Geom. Phys. 34(3-4), 287-301 (2000)

48. Ünal, B.: Doubly warped products. Differ. Geom. Appl. 15(3), 253-263 (2001)

49. Uğuz, S., Bilge, A.H.: $(3+3+2)$ warped-like product manifolds with Spin(7) holonomy. J. Geom. Phys. 61(6), 1093-1103 (2011)

50. Walsh, M.: Metrics of positive scalar curvature and generalised Morse functions. Mem. Am. Math. Soc. Part I 209(983), xviii+80 (2011)

51. Wang, Y.: Multiply warped products with a semisymmetric metric connection. Abstr. Appl. Anal. Art. ID 742371 (2014)

52. Yamabe, H.: On a deformation of Riemannian structures on compact manifolds. Osaka Math. J. 12, 21-37 (1960) 
53. Yang, K.-W.: On warped product manifolds-conformal flatness and constant scalar curvature problem. Tamkang J. Math. 29(3), 203-221 (1998)

54. Zucker, S.: $L_{2}$ cohomology of warped products and arithmetic groups. Invent. Math. 70(2), 169-218 $(1982 / 83)$

Publisher's Note Springer Nature remains neutral with regard to jurisdictional claims in published maps and institutional affiliations. 